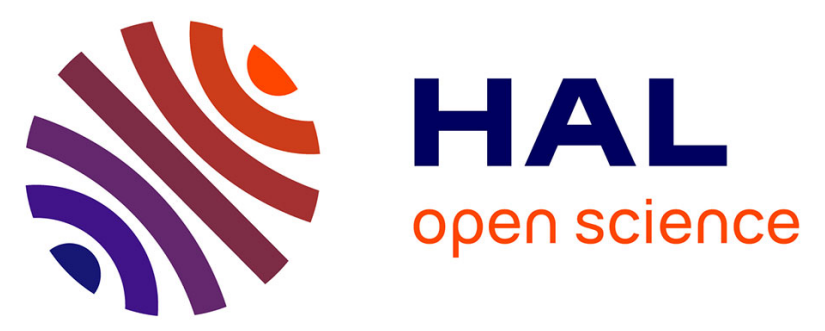

\title{
Fracture mechanics of a self-healing hydrogel with covalent and physical crosslinks: A numerical study
}

Jingyi Guo, Mincong Liu, Alan T Zehnder, Jingwen Zhao, Tetsuhara Narita, Costantino Creton, Chung-Yuen Hui

\section{- To cite this version:}

Jingyi Guo, Mincong Liu, Alan T Zehnder, Jingwen Zhao, Tetsuhara Narita, et al.. Fracture mechanics of a self-healing hydrogel with covalent and physical crosslinks: A numerical study. Journal of the Mechanics and Physics of Solids, 2018, 120, pp.79-95. 10.1016/j.jmps.2018.03.009 . hal-02074597

\section{HAL Id: hal-02074597 https://hal.science/hal-02074597}

Submitted on 21 Mar 2019

HAL is a multi-disciplinary open access archive for the deposit and dissemination of scientific research documents, whether they are published or not. The documents may come from teaching and research institutions in France or abroad, or from public or private research centers.
L'archive ouverte pluridisciplinaire HAL, est destinée au dépôt et à la diffusion de documents scientifiques de niveau recherche, publiés ou non, émanant des établissements d'enseignement et de recherche français ou étrangers, des laboratoires publics ou privés. 


\section{Fracture mechanics of a self-healing hydrogel with covalent and physical crosslinks: A numerical study ${ }^{1}$}

Jingyi Guo ${ }^{1 \$}$, Mincong Liu ${ }^{1 \$ \$}$, Alan T. Zehnder ${ }^{1}$, Jingwen Zhao ${ }^{2}$, Tetsuhara Narita ${ }^{2,3}$, Costantino Creton ${ }^{2}$, Chung-Yuen Hui ${ }^{1 \#}$

${ }^{1}$ Sibley School of Mechanical and Aerospace Engineering, Field of Theoretical \& Applied Mechanics, Cornell University, Ithaca, NY 14853

${ }^{2}$ Laboratoire Sciences et Ingénierie de la Matière Molle, PSL Research University, UPMC Univ Paris 06, ESPCI Paris, CNRS, 10 rue Vauquelin, 75231, Paris Cedex 05, France

${ }^{3}$ Global Station for Soft Matter, Global Institution for Collaborative Research and Education, Hokkaido University, Sapporo, Japan

\$,\$\$ These authors contributed equally to this work

\# Corresponding Author, ch45@cornell.edu

\section{Keywords:}

Hydrogel, fracture, finite element, crack tip field, viscoelastic, experiments

\section{Abstract}

The fracture mechanics of a stationary crack in a Poly(vinylalcohol) (PVA) hydrogel with a network consisting of chemical and physical crosslinks is studied here. Prior research by the authors has shown that the time- dependent stress strain behavior of this gel can be captured very accurately with a 3D, large deformation nonlinear viscoelastic model based on breaking kinetics of physical crosslinks. This model is used together with a novel time integration scheme to study the stress and deformation fields near the tip of a stationary crack in single edge cracked specimens. The theoretical and finite element results agree remarkably well with experimentally observed crack opening profiles. For the special case of relaxation tests exact asymptotic crack tip solutions are obtained in specimens loaded under predominantly plane stress conditions.

\section{Introduction}

Hydrogels have many applications, such as scaffolds for cells in tissue engineering ${ }^{1-2}$, artificial cartilage ${ }^{3}$ and as vehicles for drug delivery ${ }^{4}$. However, because these gels contain mostly water, they are typically very brittle and cannot be used as load bearing components in structures. The seminal work of Gong's group ${ }^{5}$ demonstrated how to make stiff yet highly extensible hydrogels with toughness rivaling that of synthetic rubber. These double network (DN) hydrogels consist of two inter-penetrating networks.

\footnotetext{
${ }^{1}$ Submitted to the Journal of the Mechanics and Physics of Solids.
} 
The first network is tightly cross-linked and brittle - the crosslinks serve as sacrificial bonds which dissipate energy. The second network is loosely crosslinked and highly extensible ${ }^{5-6}$. During loading, the first network bears most of the load and undergoes progressive damage while the second network prevents the formation and growth of macroscopic cracks $^{7-10}$. A disadvantage of these early DN gels is that the chemical crosslinks in the $1^{\text {st }}$ network cannot be reformed after failure. In the past five years, several research groups ${ }^{11-19}$ have overcome this difficulty by using sacrificial bonds that are transient, allowing them to reattach or heal after failure, see Figure 1 . These gels can be extremely tough and can recover their shapes or heal partially or completely after load removal $\left.\right|^{12,14-15,17-18}$. On the other hand, the presence of transient, or physical bonds, together with high extensibility, gives rise to highly nonlinear viscoelastic behavior. As a result, modeling the mechanical behavior of these gels when they are subjected to complex loading history remains an open problem.

Recently, we have made progress in this direction by developing a physically based, large deformation $3 D$ constitutive model which accurately predicts the nonlinear tension and torsion viscoelastic behavior of a dual-crosslink poly (vinyl alcohol) (PVA) hydrogel ${ }^{19-21}$. Briefly, this gel consists of PVA chains chemically crosslinked by glutaraldehyde and physically crosslinked by borate ions ${ }^{15-16}$. Although this gel has lower fracture toughness in comparison to those developed by Gong et al. ${ }^{14}$ and others ${ }^{13,22-23}$, it has a well-defined, simple chemical structure with only one type of transient bond having a well-defined relaxation time. In addition, the dynamics of bond breaking and healing have the unusual property of being nearly independent of strain over a wide range of strain levels. Thus the gel serves as a model system to study the interplay between bond kinetics and macroscopic mechanical behavior of the hydrogel having transient crosslinks.

While the constitutive model correctly predicts the time dependent behavior of samples loaded in uniaxial tension and torsion, it is unclear whether it can accurately capture the highly inhomogeneous state of stress near the tip of a pre-existing crack. In this paper, we address this question by comparing the loads and crack opening displacements obtained from experiments to the prediction of the model. These theoretical predictions are obtained using a 3D nonlinear finite element model of the fracture samples. Since the input material parameters to the constitutive model are completely determined by a separate tension relaxation test of an un-notched sample, there are no adjustable parameters in the fracture calculations and comparisons to experiments.

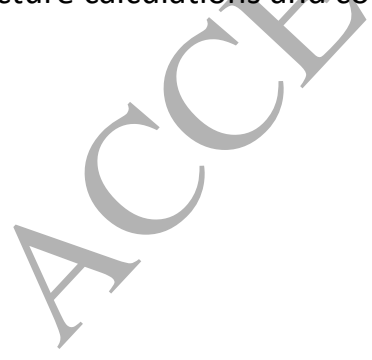



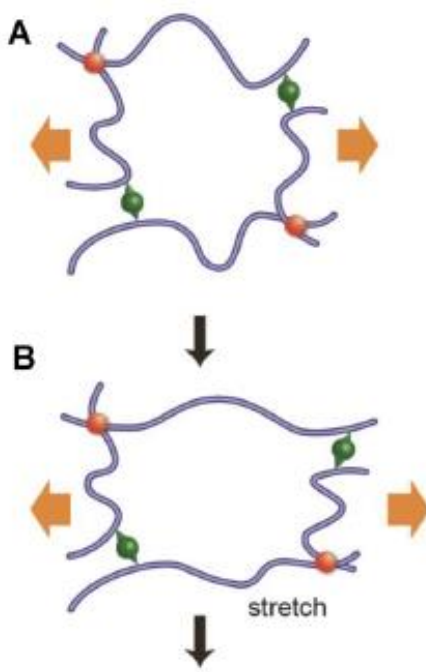

C
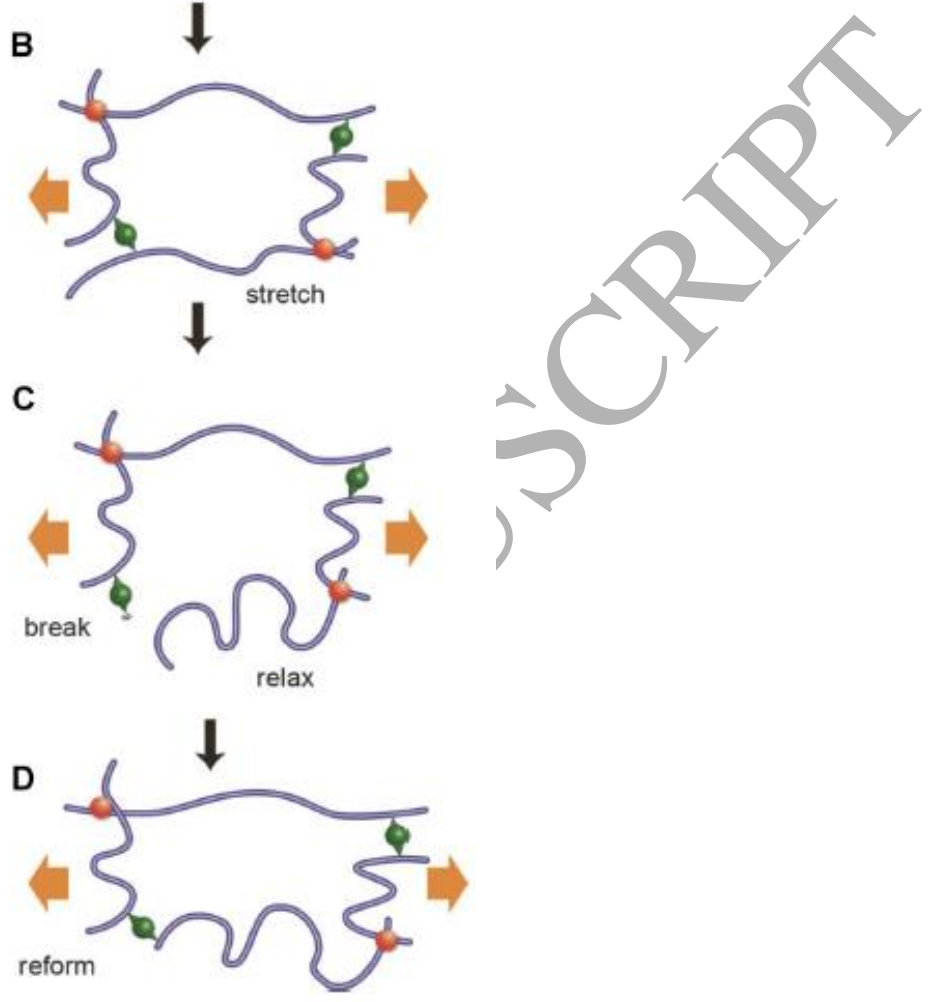

Figure 1. Breaking and healing process of physical crosslinks in dual crosslink gels and the effect on mechanical stress. From [Long et al., 2014 ACS]

A difficulty in building a computational finite element model for nonlinear viscoelastic materials is the time integration scheme. Because the stress depends on the deformation history, it may be necessary to store the entire deformation history at each node. Commercial finite element codes such as ABAQUS have built in specialized viscoelastic models with a finite number of state variables. This feature enables the use of a time integration scheme in which the stress at time $t+\Delta t$ can be computed based on its value at $t$ and the deformation gradient at $t+\Delta t$. While such models avoid the problem of storing an unmanageable amount of data, they cannot be applied to constitutive models with complex physics. Here we have developed an integration technique that resolves the difficulty with data storage while preserving the bond breaking and reforming physics of the constitutive model. 
The local damage near the crack tip is driven by stress concentration which is governed by the asymptotic behavior of the crack tip field. Although there are many studies on crack tip fields in hyperelastic solids, there are very few results on crack tip fields in nonlinear viscoelastic solids. Here we will present an exact solution of the local crack tip stress and strain fields for the special case of a relaxation test.

The plan of this paper is as follows. Section 2 is a brief review of the constitutive model. The experimental procedures are presented in Section 3. In Section 4 we examine the asymptotic behavior of the crack tip field, and an exact solution is given for the special case of a load relaxation test under plane stress conditions. Our finite element implementation of the constitutive model is presented in Section 5. Here we develop an integration scheme to bypass the storage difficulty. Comparisons of the FEM results to experiments are given in Section 6, followed by a discussion and summary in Section 7 .

\section{Constitutive Model}

The development of the constitutive model for the dual crosslink gel was detailed in previous papers ${ }^{21}$, thus only a brief summary is given here. The basic assumptions of the model are:

1) The gel network consists of permanent elastic strands attached to chemical crosslinks and temporary elastic strands attached to physical crosslinks. The chemical crosslinks are assumed to be unbreakable whereas the physical crosslinks can break and reform.

2) The strain energy of the gel network is equal to the sum of that carried by each individual polymer strand.

3) The breaking and reforming of physical crosslinks achieves dynamic equilibrium quickly after the gel is synthesized. This dynamic equilibrium results in a constant molar fraction of connected and broken temporary strands and a constant reforming rate, which is denoted by $\bar{\gamma}_{\infty}$.

4) The stress sustained by a temporary strand is instantaneously relaxed when it breaks. Immediately after a temporary strand is reattached, it is in a relaxed state and carries no strain energy. The deformation of a temporary chain reconnected at time $\tau$ is described by the deformation gradient tensor $\mathbf{F}^{\tau \rightarrow t}$. The superscript $\tau \rightarrow t$ indicates that the temporary strand experiences the deformation history from its birth (reattachment) at $\tau$ to the current time $t$.

5) Macroscopically the gel is assumed to be incompressible and isotropic. When subjected to the same stress, the chains between physical and chemical crosslinks deform in the same way so that the same strain energy model applies to permanent and temporary strands. In the following, the strain energy of the undamaged network $W_{0}$ is assumed to depend only on the first invariant of the right Cauchy-Green tensor, $I=\operatorname{tr}\left[\left(\mathbf{F}^{0 \rightarrow t}\right)^{\mathrm{T}} \mathbf{F}^{0 \rightarrow t}\right]$.

6) The rates of strand breaking and reattaching are independent of the imposed strain history, as suggested by experimental findings ${ }^{16,21}$

With these assumptions, the nominal stress tensor $\mathbf{P}$ and true stress tensors $\boldsymbol{\sigma}$ are, respectively: 


$$
\begin{aligned}
& \mathbf{P}=-p\left(\mathbf{F}^{0 \rightarrow t}\right)^{-T}+\left.2[\rho+n(t)] \frac{d W_{0}}{d I}\right|_{I=I(\boldsymbol{x}, t)} \mathbf{F}^{0 \rightarrow t}+\left.2 \bar{\gamma}_{\infty} \int_{0}^{t} \phi_{B}\left(\frac{t-\tau}{t_{B}}\right) \frac{d W_{0}}{d I}\right|_{I=H(\boldsymbol{x}, \tau, t)} \mathbf{F}^{\tau \rightarrow t}\left(\mathbf{F}^{0 \rightarrow \tau}\right)^{-\mathrm{T}} d \tau \\
& \boldsymbol{\sigma}=-p \boldsymbol{I}+\left.2[\rho+n(t)] \frac{d W_{0}}{d I}\right|_{I=I(\boldsymbol{x}, t)} \mathbf{B}^{0 \rightarrow t}+2 \bar{\gamma}_{\infty}\left[\left.\int_{0}^{t} \phi_{B}\left(\frac{t-\tau}{t_{B}}\right) \frac{d W_{0}}{d I}\right|_{I=H(\boldsymbol{x}, \tau, t)} \mathbf{F}^{\tau \rightarrow t}\left(\mathbf{F}^{0 \rightarrow \tau}\right)^{-\mathrm{T}} d \tau\right]\left(\mathbf{F}^{0 \rightarrow t}\right)^{\mathrm{T}}
\end{aligned}
$$

where $\mathbf{B}^{0 \rightarrow t}=\mathbf{F}^{0 \rightarrow t}\left(\mathbf{F}^{0 \rightarrow t}\right)^{\mathrm{T}}, p$ is the pressure that enforces the incompressibility constraint, $\rho$ is the molar fraction of the permanent chains to the total number of chains; $W_{0}(I)$ is the strain energy per unit reference volume of the undamaged network, and

$$
\mathrm{H}(\boldsymbol{x}, \tau, t)=\operatorname{tr}\left[\left(\mathbf{F}^{\tau \rightarrow t}\right)^{\mathrm{T}} \mathbf{F}^{\tau \rightarrow t}\right]
$$

The function in (1a)

$$
\phi_{B}\left(\frac{t}{t_{B}}\right)=\left(1+\left(\alpha_{B}-1\right) \frac{t}{t_{B}}\right)^{\frac{1}{1-\alpha_{B}}}
$$

denotes the probability of breaking of physical crosslinks, where $t_{B}$ is the characteristic time for breaking; $2>\alpha_{B}>1$ is a material constant that specifies the rate of decay of $\phi_{B}$. Finally, $n(t)$, the fraction of physical bonds present at $t=0$ and still attached at $t$ is

$$
n(t) \equiv \bar{\gamma}_{\infty} \int_{-\infty}^{0} \phi_{B}\left((t-\tau) / t_{B}\right) d \tau=\bar{\gamma}_{\infty} \frac{t_{B}}{2-\alpha_{B}}\left(1+\left(\alpha_{B}-1\right) \frac{t}{t_{B}}\right)^{\frac{2-\alpha_{B}}{1-\alpha_{B}}}
$$

Note that $n(t)$ is a decaying function of time since $2>\alpha_{B}>1$. Equations (1-4) assume that deformation is identically zero for time $t<0$. For uniaxial tension, equation (1) reduces to

$$
\mathbf{P}_{11}=\left.2(\rho+n(t)) \frac{d W_{0}}{d I}\right|_{I(t)}\left[\lambda(t)-\frac{1}{\lambda^{2}(t)}\right]+\left.2 \bar{\gamma}_{\infty} \int_{0}^{t} \phi_{B}\left(\frac{t-\tau}{t_{B}}\right) \frac{d W_{0}}{d I}\right|_{H(\tau, t)}\left[\frac{\lambda(t)}{\lambda^{2}(\tau)}-\frac{\lambda(\tau)}{\lambda^{2}(t)}\right] d \tau
$$

where $\lambda$ is the stretch ratio in the loading direction, and

$$
I(t)=\lambda^{2}(t)+\frac{2}{\lambda(t)}, H(\tau, t)=[\lambda(t) / \lambda(\tau)]^{2}+2 \lambda(\tau) / \lambda(t), \quad \tau<t
$$


The first term on the right hand side of eq (5) describes the loss of stress due to breaking of the original temporary strands while the integral term describes the recovery of stress from the reattachment of temporary strands.

For the rest of this paper, all chains are assumed to be Gaussian, so $W_{0}$ is given by the neo-Hookean model:

$$
W_{0}=\frac{\mu}{2}(I-3)
$$

where $\mu$ is the small strain shear modulus of the neo-Hookean model at $t=0$ when no load is applied and all the physical strands remain intact. For this case, the model is completely specified by four independent material parameters, $\mu \rho, \mu \bar{\gamma}_{\infty}, \alpha_{B}, t_{B}$. The parameter $\mu \rho$ is the long time modulus of the gel in a relaxation test when the physical strands do not carry any load. It corresponds to the shear modulus of the chemically crosslinked network. Since $\bar{\gamma}_{\infty}$ is the equilibrium rate of reformation of the temporary crosslinks, $\mu \bar{\gamma}_{\infty}$ can be roughly thought of as a parameter which measures how stresses increases with the number of healed strands per unit time.

To determine the four material parameters, we first carry out a uniaxial tensile relaxation test where the sample is stretched under constant rate to a fixed stretch ratio, then held at this stretch until the stress reaches a plateau. The plateau stress, the log - log plot of the stress vs time at large time (we use both the slope and the intercept from the linear fit), and the initial slope of the loading part provide us four conditions for the four material parameters, so that the material parameters can be calculated. Then the parameters can be iterated by hand to give the best fit to loading/unloading tests carried out at constant stretch rates. Further details of the model identification procedure are described in our previous work $^{19-21}$.

Previous work by the authors has shown that this model accurately predicts the stress and strain behavior in uniaxial tension and torsion tests ${ }^{19-21}$. The uniaxial tests involve loading and unloading at different stretch rates, covering at least three decades of stretch rates. Since the properties of samples vary slightly from batch to batch the sets of loading and unloading tests are repeated here. Results of these tests, given in the supplementary information, are used to determine the model parameters.

As an example, the nominal stress versus stretch from a cyclic test is plotted in Figure 2 . Here the sample is loaded with a constant stretch rate of $\dot{\lambda}=0.01 / \mathrm{s}$. At $\lambda=1.3$, the sample is unloaded to zero stress at an unloading rate of $0.01 / \mathrm{s}$. The experimental data is the solid line, the prediction of eqn. (5) is plotted as a dotted line. The agreement between experiment and theory is striking. More results for loading/unloading at different stretch rates can be found in the supplementary information. 

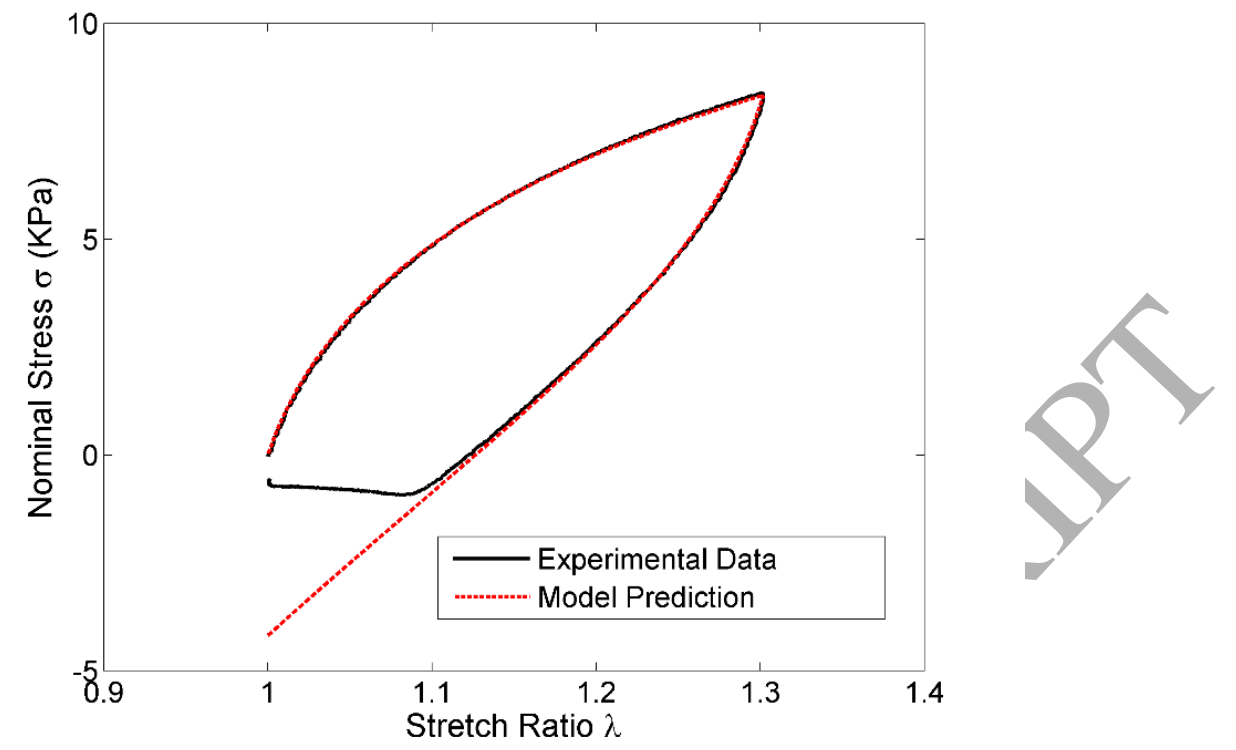

Figure 2. Stress vs. stretch for a loading/unloading cycle to $\lambda=1.3$ at loading/unloading stretch rate of $0.01 / \mathrm{s}$. In the experiments, upon unloading the sample goes into compression then buckles. The model calculation does not account for buckling

\section{Experiments}

\subsection{Material preparation}

The material in this study is poly (vinyl alcohol) (PVA) hydrogel crosslinked by both permanent covalent bonds and reversible physical bonds. Details of synthesis were given in previous works ${ }^{16}$. Here we briefly summarize the procedure. The chemically crosslinked gel was made by first mixing glutaraldehyde solution into PVA solution at $\mathrm{pH}=1.4$. The PVA concentration in the solution was $12 \mathrm{wt}$. \% and the molar ratio of chemical cross-linker and PVA monomers was 1:500. The solution was then injected into a mold consisting of two acrylic plates spaced a fixed distance apart by rubber sheets that also serve to seal the mold. After 24 hours, the chemically crosslinked gel was removed from the mold and washed with plenty of water to neutral $\mathrm{pH}$. Then the chemical gel was soaked in a $\mathrm{NaCl} / \mathrm{Borax}$ solution (Borax, $1 \mathrm{mmol} / \mathrm{L} ; \mathrm{NaCl} 90 \mathrm{mmol} / \mathrm{L}$ ) for 3 days. The infusion of the ionic solution causes the physical bonds to form. Once the $\mathrm{NaCl} /$ Borax soak was complete the samples were stored in the same solution for up to two weeks prior to testing.

\subsection{Uniaxial tension tests for model identification}

The material parameters for the constitutive model are determined by a series of uniaxial tensile tests. All uniaxial tensile tests were performed using samples that are $12 \mathrm{~mm}$ wide and $1.3 \mathrm{~mm}$ thick. The tests were performed using the custom built tensile tester shown in Figure 3. Load was measured by an Interface SMT1-1.1 load cell (4.9 N capacity) and displacement by an OMEGA LD620 LVDT. Translational motion was provided by a Zaber X-LSM200A-E03 translational stage. The stage is driven by a two-phase 
stepper motor. The linear motion resolution of the stage is $0.047625 \mu \mathrm{m}$. The load and displacement signals were recorded using a Keithley Model 2701 multiplexing digital voltmeter at rates of 25 pairs of data/sec. Samples were gripped using sandpaper lined grips as shown in Figure $3 b$ and using a gauge length of $24 \mathrm{~mm}$. A certain amount of trial and error is required to apply enough pressure on the grips to prevent slippage while not overly tightening which would cause early failure of the sample at the grips. The hydrogel samples were tested while immersed in mineral oil to prevent drying. The oil exerts a buoyant force on the grips. This force was calibrated experimentally and subtracted from the measured load to avoid systematic error.

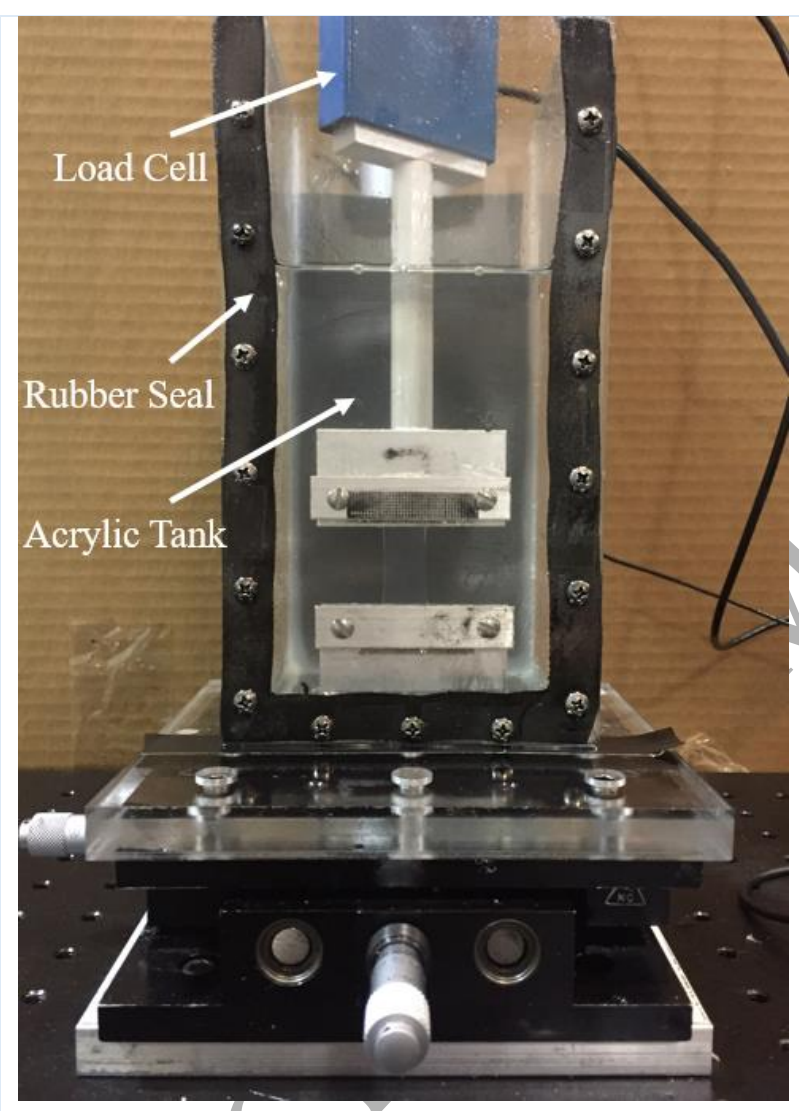

(a)

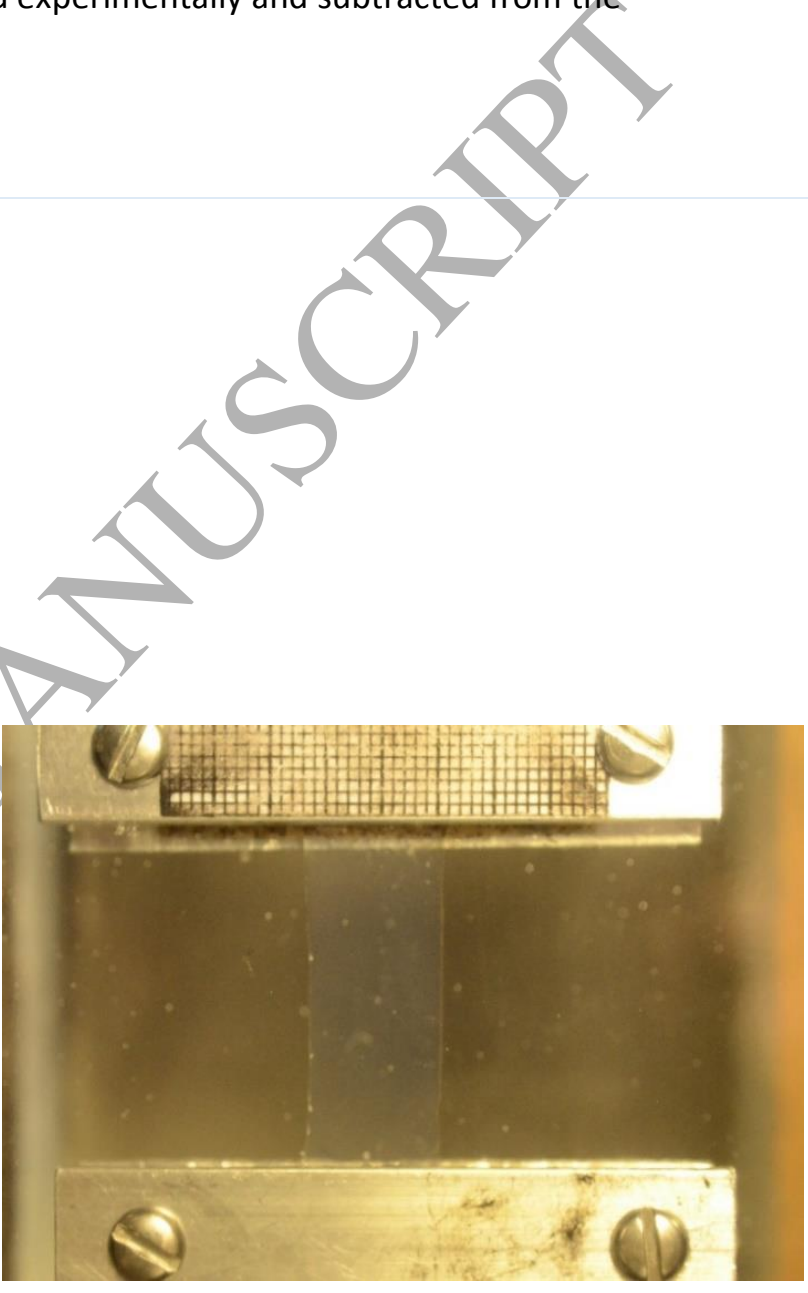

(b)

Figure 3. Experimental setup. (a) Overview showing load cell (blue), acrylic tank and rubber seal filled with mineraloil. Translation stage at the bottom of the setup allows the lower grip to be aligned with the upper grid. The tip of the LVDT is seen at the top pressing against the load cell mounting block. (b) Close up of a typical tension sample held in place with sandpaper lined grips.

Previously, we have shown that all four material parameters in our model can be estimated by a single stress relaxation test ${ }^{21}$. In this test, a sample is loaded to a stretch ratio of 1.3 at a fast loading rate 
$(0.5 / \mathrm{s})$ and then held at this stretch for 30 minutes to obtain the stress response vs. time. An initial parameter set is estimated by fitting the relaxation data. The parameters are then used to predict the stress history of samples loaded at a constant stretch rate to a maximum stretch of 1.3 and then unloaded to its original length at two different loading/unloading rates, $0.005 / \mathrm{s}$ and $0.01 / \mathrm{s}$. Here the nominal stretch rate is defined as the loading speed divided by the initial gauge length of the sample. $A$ series of such calculations are performed, iterating the model parameters from the initial estimate until a satisfactory fit is obtained. As Figure 2 shows we are able to obtain an excellent fit of the model to the measured data. Note that material properties will vary slightly from batch to batch, thus we repeat the characterization tests and fitting for each new batch of hydrogel prepared. As the data presented in this paper come from different gel batches, the model parameters corresponding to each batch are noted in the supplemental information.

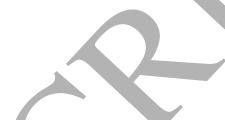

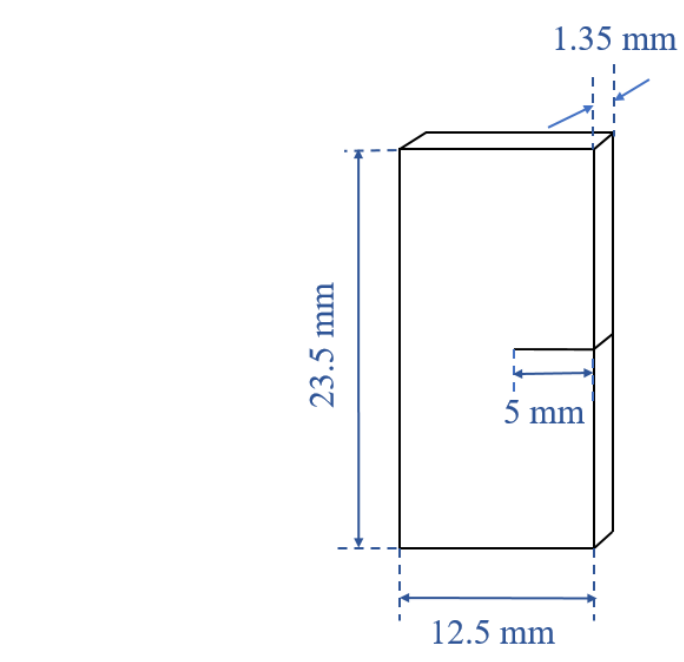

Geometry A

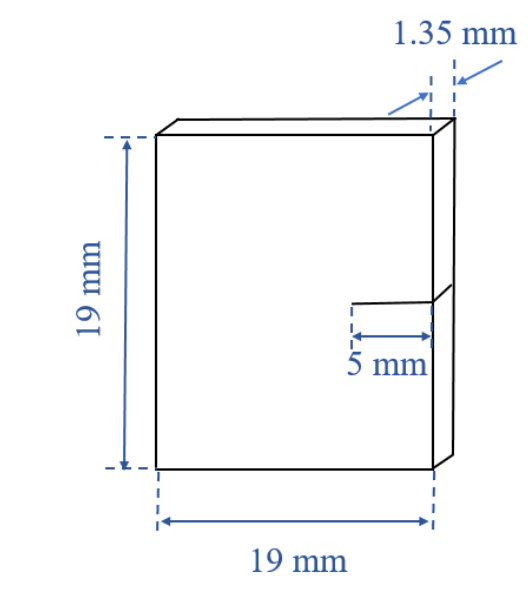

$19 \mathrm{~mm}$

Geometry B

Figure 4. Geometry used for fracture experiments.

\subsection{Fracture tests}

Mode I fracture tests were performed using edge-notched samples with two different geometries, $A$ and $B$, as shown in Figure 4. The dimensions of sample A were: $12.5 \mathrm{~mm}$ (width), $1.35 \mathrm{~mm}$ (thickness) and $23.5 \mathrm{~mm}$ (gauge length). The dimensions of sample B were: $19 \mathrm{~mm}$ (width), $1.35 \mathrm{~mm}$ (thickness) and 19 $\mathrm{mm}$ (gauge length)= An initial edge crack of length of $5 \mathrm{~mm}$ was cut in all samples. The $A$ samples were loaded to failure at six nominal stretch rates of $0.001 / \mathrm{s}, 0.003 / \mathrm{s}, 0.01 / \mathrm{s}, 0.03 / \mathrm{s}, 0.1 / \mathrm{s}$ and $0.3 / \mathrm{s}$. The $B$ samples were loaded at a single applied stretch rate of $0.01 / \mathrm{s}$. For each set of geometry and loading rate, the tests were repeated at least 3 times.
} 


\section{Asymptotic Crack Tip Fields for Relaxation Test}

In the following, we consider a relaxation test where a notched specimen is subjected to a step displacement at $t=0$. We derive the first term of the resulting asymptotic crack tip stress and displacement fields. The loading and specimen geometry are such that the crack is loaded in Mode I. Since the applied displacement is fixed for all $t>0$, the deformation gradient at any point is independent of time, that is,

$$
\mathbf{F}^{\tau \rightarrow t}=\mathbf{I} \quad \tau>0, \quad \mathbf{F}^{0 \rightarrow \tau}=\mathbf{F}^{0 \rightarrow t} .
$$

Justification of this assumption is given in the Appendix. Using (7-8), the true stress is given by:

$$
\boldsymbol{\sigma}=-p^{*} \mathbf{I}+\mu[\rho+n(t)] \mathbf{B}^{0 \rightarrow t}
$$

where $p^{*} \equiv p-\mu \bar{\gamma}_{\infty} \int_{0}^{t} \phi_{B}\left((t-\tau) / t_{B}\right) d \tau$. Since $p$ is an undetermined pressure fjeld, we can treat (9) as an incompressible neo-Hookean solid with a small strain shear modulus $[\rho+n(t)] \mu$ that depends on time. Therefore, the asymptotic behavior of the stress field of a Mode I crack during relaxation is identical to that of a Mode I crack in an incompressible neo-Hookean solid with a time dependent shear modulus. Since thin samples are typically used to study fracture, we consider plane stress, which we later show is a good model to describe the crack tip field. Using a result due to Geubelle and Knauss ${ }^{25}$, the true stress as $r \rightarrow 0$ is given by:

$$
\sigma_{11}=\mu[\rho+n(t)] c^{2}, \quad \sigma_{12}=\sigma_{21}=-\frac{\mu[\rho+n(t)]}{2 \sqrt{r}} a c \sin (\theta / 2), \quad \sigma_{22}=\frac{\mu[\rho+n(t)]}{4 r} a^{2}
$$

where $a$ and $c$ are time independent constants that depend on the applied displacement and the specimen geometry. They are similar to stress intensity factors in linear elastic fracture mechanics and can be determined by finite element calculation. In (10), $(r, \theta)$ is a polar coordinate system located at the crack tip in the reference configuration (see Figure 5). A consequence of (10) is that the true stresses decay with time as:

$$
\sigma_{11}^{\sigma} / \sigma_{11}^{0}=\sigma_{12} / \sigma_{12}^{0}=\sigma_{22} / \sigma_{22}^{0}=[\rho+n(t)] /[\rho+n(0)]
$$

where $\sigma_{\alpha \beta}^{0}$ denote the true stresses at $t=0^{+}$. The crack opening displacement $u_{2}$ does not change with time in a perfect relaxation test (where the displacement is achieved instantaneously). Near the crack tip, it is given by ${ }^{24}$

$$
u_{2}(r \rightarrow 0, \theta=\pi)=a \sqrt{r},
$$

Our theory can be tested by comparing the crack opening displacement based on finite element model (FEM) (details are given in Section 5) to that observed in a relaxation test. In this test, the specimen was 
first stretched at a constant rate of $0.03 / \mathrm{s}$ to a stretch ratio of 1.3. This displacement was fixed after the $10 \mathrm{~s}$ loading period, after which the measured load starts to relax. Figure 6 compares the load relaxation curve obtained from this experiment and from FEM. In addition, since in a perfect relaxation test each stress component depends only on $\rho+n(t)$, so should the load measured by the load cell. Thus the result from a perfect relaxation test is also plotted in Figure 6 , and it matches both the experimental and FEM results for times about $10 \mathrm{~s}$ past the loading period. The discrepancy for $\mathrm{t}<10 \mathrm{~s}$ is due to the fact that theory assumes an infinitely fast loading rate which is impossible to carry out in practice. The crack opening displacement obtained using FEM is plotted in Figure 7. Near the crack tip, its behavior agrees with (11b) and the normalized crack tip displacement parameter is found to be $a^{*} \equiv a / \sqrt{L}=0.457$. We also plot $\sigma_{22}(t)$ at $t=496 \mathrm{~s}$ (end of the test) from the FEM calculation and compare this to analytical results from equation (10) with $a^{*}=0.457$.

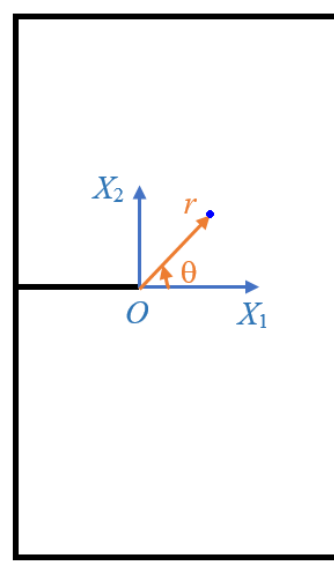

Figure 5. A Mode I plane stress crack sample in reference (undeformed) configuration. 


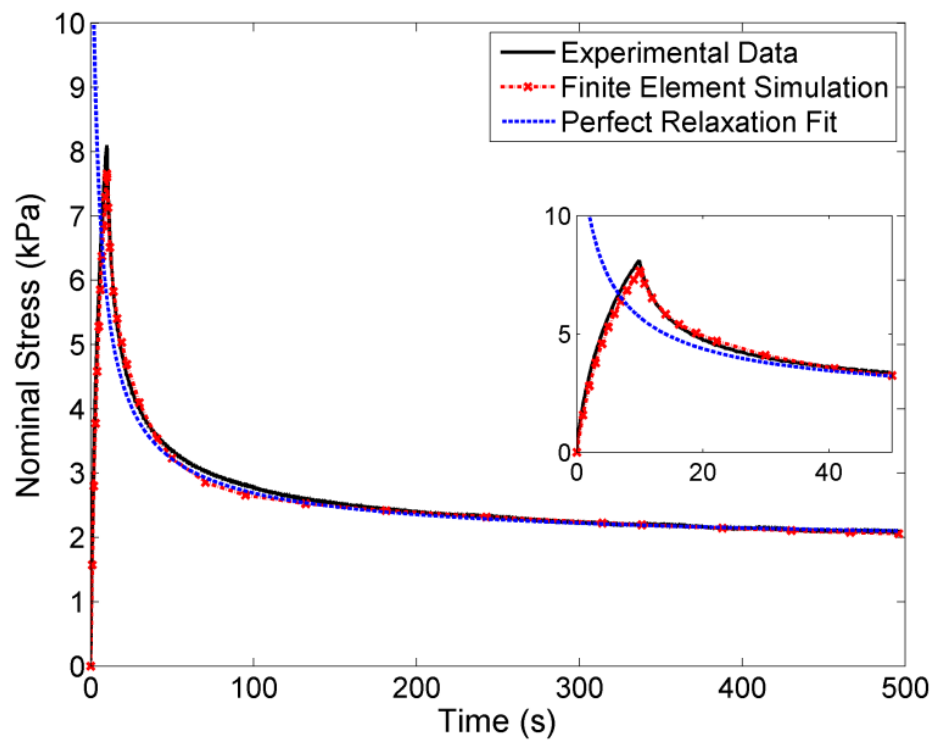

Figure 6. Nominal stress (load on top surface divided by initial uncracked area) in a relaxation test with a loading period of $10 \mathrm{~s}$ and stretch ratio 1.3. The black curve is experimental data and the red dashed curve is FEM results. Response from a perfect relaxation test (proportional to $\rho+n(t)$ ) is plotted in blue dashed curve.

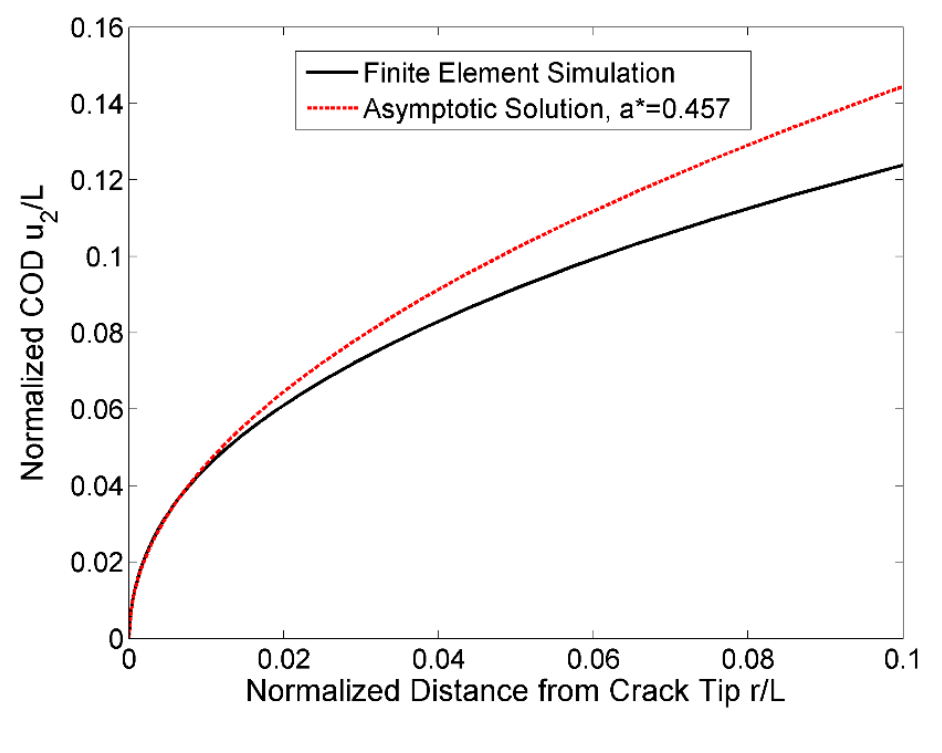

Figure 7. Crack opening displacement of the relaxation test compared with asymptotic solution. The normalized crack tip displacement parameter obtained from (11) is $a^{*} \equiv a / \sqrt{L}=0.457$. Lengths are normalized by the uncracked sample width $L$. 


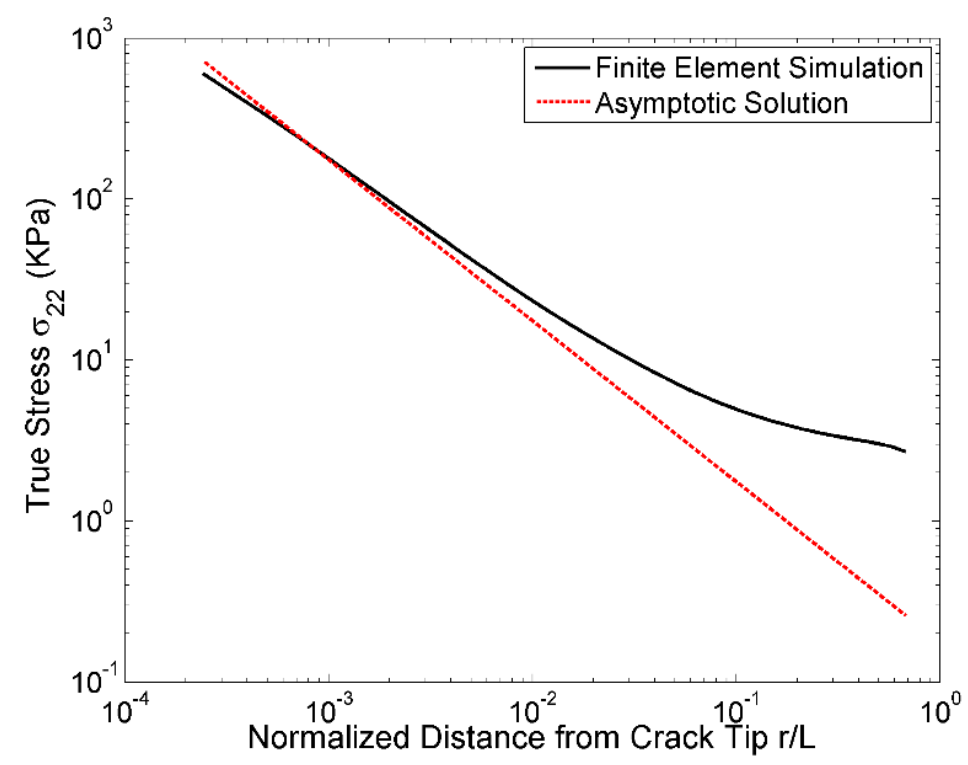

Figure 8. True stress $\sigma_{22}(t=496 s)$ in front of the crack tip. Lengths are normalized by the uncracked sample width $L$.

\section{Finite Element Implementation of Constitutive Model}

A 3D finite element model will be used to study the crack tip fields corresponding to the experiments described in section 3 . Although a constitutive model that fully depicts the material behavior is already developed, it is not trivial to implement this model into any existing commercial finite element code. We have chosen to implement our model in ABAQUS. The two major difficulties with this implementation are:

1) The constitutive model is not in ABAQUS's material library ${ }^{25}$, nor can it be approximated by any of the existing models, thus it is necessary to write a UMAT (user material subroutine);

2) The evolution of the integral term in stress requires the storage of all deformation gradient history for each node. Since there are about $10^{5}$ nodes in a typical simulation, and the number of time steps is on the order of $10^{3}$, the memory requirement is too large.

To address the second difficulty, let us denote the integral term in the Cauchy stress $\sigma$ by $\mathbf{T}(t) \equiv \int_{0}^{t} \phi_{B}(t-\tau) \mathbf{F}^{\tau \rightarrow t}\left(\mathbf{F}^{\tau \rightarrow t}\right)^{\mathrm{T}} d \tau$. Assume that $\mathbf{T}(t)$ and the increment in deformation $\mathbf{F}^{t \rightarrow t+\Delta t}$ are known at $t$, which is the case for ABAQUS. We want to compute the increment in $\mathbf{T}(t)$ after a time step $\Delta t$, i.e., 


$$
\begin{aligned}
\Delta \mathbf{T} & =\mathbf{T}(t+\Delta t)-\mathbf{T}(t) \\
& =\int_{0}^{t+\Delta t} \phi_{B}(t+\Delta t-\tau) \mathbf{F}^{\tau \rightarrow t+\Delta t}\left(\mathbf{F}^{\tau \rightarrow t+\Delta t}\right)^{\mathrm{T}} d \tau-\int_{0}^{t} \phi_{B}(t-\tau) \mathbf{F}^{\tau \rightarrow t}\left(\mathbf{F}^{\tau \rightarrow t}\right)^{\mathrm{T}} d \tau
\end{aligned}
$$

However, the LHS of (12) cannot be obtained as a function of just $\mathbf{T}(t)$ and $\mathbf{F}^{t \rightarrow t+\Delta t}$ for our power law breaking function $\phi_{B}(t)$ (see (3)). To update $\mathbf{T}(t)$ we need to know $\mathbf{F}^{\tau \rightarrow t}$ at all $\tau \leq t$.

The second difficulty can be circumvented by replacing the power law breaking function $\phi_{B}(t)$ by a Prony series of $N$ terms. (As a rule of thumb, good fits can generally be attained using one term for each decade of time):

$$
\phi_{B}(t)=\left[1+\left(\alpha_{B}-1\right)\left(t / t_{B}\right)\right]^{\frac{1}{1-\alpha_{B}}} \rightarrow \sum_{i=1}^{N} g_{i} e^{-\frac{t}{\tau_{i}}}
$$

where $g_{i}$ and $t_{i}$ are constants related to $\alpha_{B}$ and $t_{B}$. Once we make the replacement, the integral term in Cauchy stress $\mathbf{T}(t)$ can be updated using only current information. The proof is as follows:

$$
\begin{aligned}
\mathbf{T}(t)=\sum_{i=1}^{N} \mathbf{T}_{\mathbf{i}}(t) & \equiv \sum_{i=1}^{N} g_{i} \int_{0}^{t} e^{-\frac{t-\tau}{\tau_{i}}} \mathbf{F}^{\tau \rightarrow t}\left(\mathbf{F}^{\tau \rightarrow t}\right)^{\mathrm{T}} d \tau \\
\mathbf{T}_{\mathbf{i}}(t+\Delta t) & =g_{i} \int_{0}^{t+\Delta t} e^{-\frac{t+\Delta t-\tau}{\tau_{i}}} \mathbf{F}^{\tau \rightarrow t+\Delta t}\left(\mathbf{F}^{\tau \rightarrow t+\Delta t}\right)^{\mathrm{T}} d \tau \\
& =g_{i} e^{-\frac{\Delta t}{\tau_{i}}} \mathbf{F}^{t \rightarrow t+\Delta t} \cdot \int_{0}^{t} e^{-\frac{t-\tau}{\tau_{i}}} \mathbf{F}^{\tau \rightarrow t}\left(\mathbf{F}^{\tau \rightarrow t}\right)^{\mathrm{T}} d \tau \cdot\left(\mathbf{F}^{t \rightarrow t+\Delta t}\right)^{\mathrm{T}}+g_{i} \int_{t}^{t+\Delta t} e^{-\frac{t+\Delta t-\tau}{\tau_{i}}} \mathbf{F}^{\tau \rightarrow t+\Delta t}\left(\mathbf{F}^{\tau \rightarrow t+\Delta t}\right)^{\mathrm{T}} d \tau \\
& =g_{i} e^{-\frac{\Delta t}{\tau_{i}}} \mathbf{F}^{t \rightarrow t+\Delta t} \cdot \mathbf{T}_{\mathbf{i}}(t) \cdot\left(\mathbf{F}^{t \rightarrow t+\Delta t}\right)^{\mathrm{T}}+g_{i} \int_{t}^{t+\Delta t} e^{-\frac{t+\Delta t-\tau}{\tau_{i}}} \mathbf{F}^{\tau \rightarrow t+\Delta t}\left(\mathbf{F}^{\tau \rightarrow t+\Delta t}\right)^{\mathrm{T}} d \tau
\end{aligned}
$$

The second term in (14) can be evaluated using trapezoidal rule. This results in

$$
\mathbf{T}_{\mathbf{i}}(t+\Delta t)=g_{i} e^{-\frac{\Delta t}{\tau_{i}}} \mathbf{F}^{t \rightarrow t+\Delta t} \cdot \mathbf{T}_{\mathbf{i}}(t) \cdot\left(\mathbf{F}^{t \rightarrow t+\Delta t}\right)^{\mathrm{T}}+\frac{1}{2} g_{i}\left[e^{-\frac{\Delta t}{\tau_{i}}} \mathbf{F}^{t \rightarrow t+\Delta t}\left(\mathbf{F}^{t \rightarrow t+\Delta t}\right)^{\mathrm{T}}+\mathbf{I}\right]
$$

Thus, each term in $\mathbf{T}(t)$ depends only on $\mathbf{T}_{\mathbf{i}}(t)$ and $\mathbf{F}^{t \rightarrow t+\Delta t}$.

The next step is to develop a scheme to select the unknown coefficients in the series (13). Ideally, we would like a small number of terms. We use the following idea: For a given function $G(s)$, we assume that it can be expanded in an infinite series of the form:

$$
G(s)=\sum_{i} g_{i} e^{-\frac{s}{\tau_{i}}}=\sum_{i} g_{i} e^{-s \zeta_{i}}, \quad \text { where } \zeta_{i} \equiv \tau_{i}^{-1},
$$


We seek a function $\hat{g}(\zeta)$ such that

$$
G(s)=\sum_{i=1}^{\infty} g_{i} e^{-s \zeta_{i}}=\int_{0}^{\infty} \hat{g}(\zeta) e^{-s \zeta} d \zeta
$$

Thus, $G(s)$ is the Laplace transform of $\hat{g}(\zeta)$. To approximate $G(s)$ by a finite number of terms in the series, as outlined in Figure 9, we divide $[0, \infty]$ into $N$ intervals such that each inverse characteristic time, $\zeta_{i}$, lies in an interval, i.e. $\zeta_{i} \in\left[\zeta_{i 0}, \zeta_{i 0}+\Delta \zeta_{i}\right], 1 \leq i \leq N\left(\zeta_{(i+1) 0} \equiv \zeta_{i 0}+\Delta \zeta_{i}\right)$, with $\zeta_{10}=0$ and $\zeta_{j 0}<\zeta_{i 0}, j<i$. Based on mean value theorem of calculus, there exists a point $\zeta_{i} \in\left[\zeta_{i 0}, \zeta_{i 0}+\Delta \zeta_{i}\right]$ such that

$$
g_{i} \equiv \hat{g}\left(\zeta_{i}\right) \Delta \zeta_{i}=\int_{\zeta_{i 0}}^{\zeta_{i 0}+\Delta \zeta_{i}} \hat{g}(\zeta) d \zeta
$$

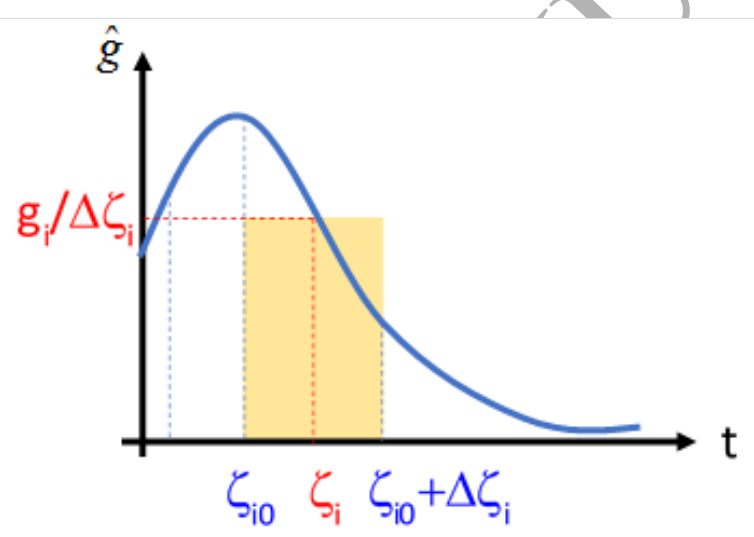

Figure 9. Finding the average value and average point of function $\hat{g}(\zeta)$.

That is, $\zeta_{i} \in\left[\zeta_{i 0}, \zeta_{i 0}+\Delta \zeta_{i}\right]$ is the inverse characteristic time, and the average value of the function $\hat{g}\left(\zeta_{i}\right)$ multiplied by the length of the interval $\Delta \zeta_{i}$ is the Prony coefficient $g_{i}$. In doing this, we ensure $G(s=0)=\int_{0}^{\infty} \hat{g}(\zeta) d \zeta=\sum_{i=1}^{N} g_{i}$.

For our case, instead of approximating the 'real' breaking function $\phi_{B}(t)$, we fit the integral of the breaking function, $n(t)$, i.e., 


$$
G(s) \equiv\left[1+\left(\alpha_{B}-1\right)\left(s / t_{B}\right)\right]^{\frac{2-\alpha_{B}}{1-\alpha_{B}}}=\sum_{i=1}^{4} g_{i} e^{-\frac{s}{\tau_{i}}}
$$

This is because the breaking function always appears in an integral, either as $n(t)$ or multiplied by the deformation gradient and then being integrated; the physical meaning behind is that the total number of bonds that survive (rather than the survivability) matters. Thus it is important to get this integral correct. On the other hand, because the integral of $\phi_{B}(t)$ is approximated by a Prony series, so is $\phi_{B}(t)$ itself, so the argument above still stands true.

We pick the number of terms in the Prony series to be 4 since we our experiments usually lasted less than 1000 seconds, so the time intervals $\left[1 / \zeta_{i 0}, 1 /\left(\zeta_{i 0}+\Delta \zeta_{i 0}\right)\right]$ are [0, 1s], [1s, 10s], [10s, 100s], [100s, 1000s] respectively. The inverse Laplace transform of $G(s) \equiv\left[1+\left(\alpha_{B}-1\right)\left(s / t_{B}\right)\right]^{\frac{2-\alpha_{B}}{1-\alpha_{B}}}$ is

$$
\hat{g}(\zeta)=\frac{\left(\frac{\alpha_{B}-1}{t_{B}}\right)^{\frac{2-\alpha_{B}}{1-\alpha_{B}}}}{\Gamma\left(\frac{2-\alpha_{B}}{\alpha_{B}-1}\right)} \zeta^{-\frac{3-2 \alpha_{B}}{1-\alpha_{B}}} \exp \left(-\frac{t_{B}}{\alpha_{B}-1} \zeta\right) .
$$

where $\Gamma$ is the Gamma function. We now use (18) to determine the Prony coefficients $g_{i}$ and the characteristic times $\tau_{i}=\zeta_{i}^{-1}$. Since this set of $\left(\tau_{i}, g_{i}\right)$ s match only $n(t=0)$, one can make slight adjustments to optimize the fit. In the fitting process, once we obtain this set of $\left(\tau_{i}, g_{i}\right)$ s, we fix the $\tau_{i} \mathrm{~s}$ but modify the $g_{i}$ s manually to obtain an optimized fit of $n(t)$ throughout the time history.

Figures $10 a, b$ shows fitting of the integral of the breaking function, $n(t)$ and of a uniaxial tension test. With $N=4$ terms, excellent fits of both the breaking function and of the stress in uniaxial tension are obtained. The prediction using the original power law breaking model is also shown in the same figure as a comparison. This figure confirms the accuracy of the Prony series approximation as well as our UMAT implementation. We next use this tool to study the stress and displacement field of a cracked sample. The comparison of simulation to experimental results is discussed in the next section. 


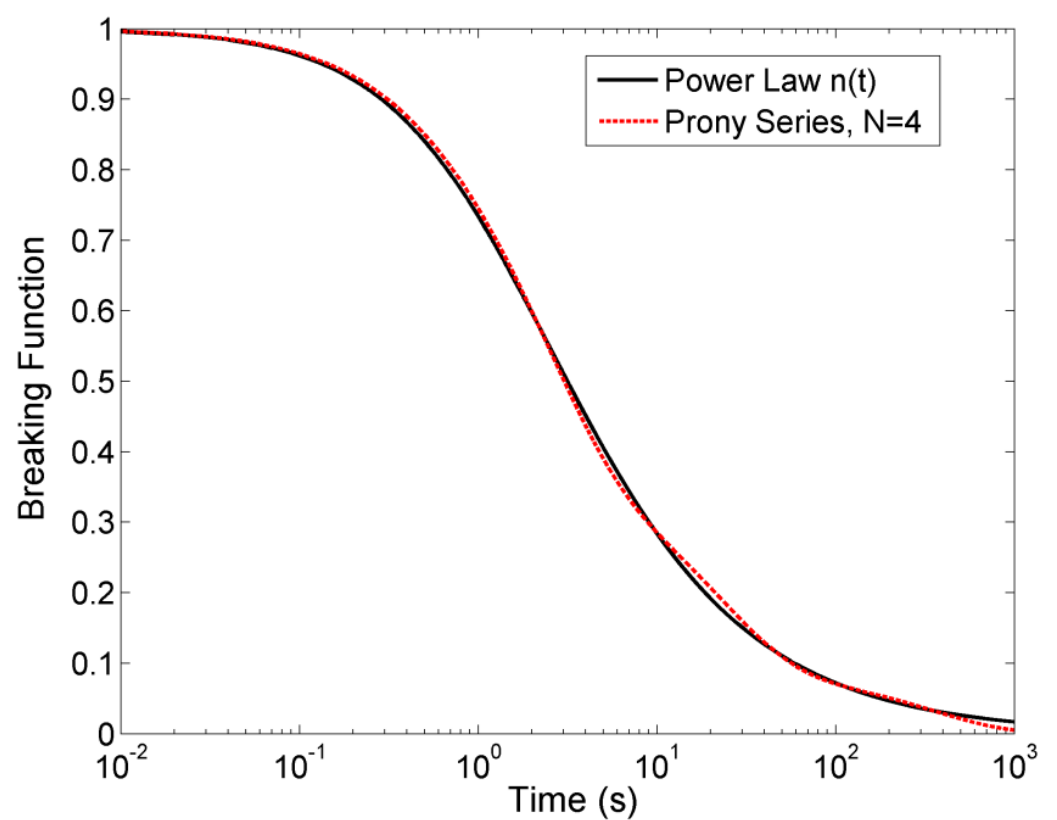

(a)

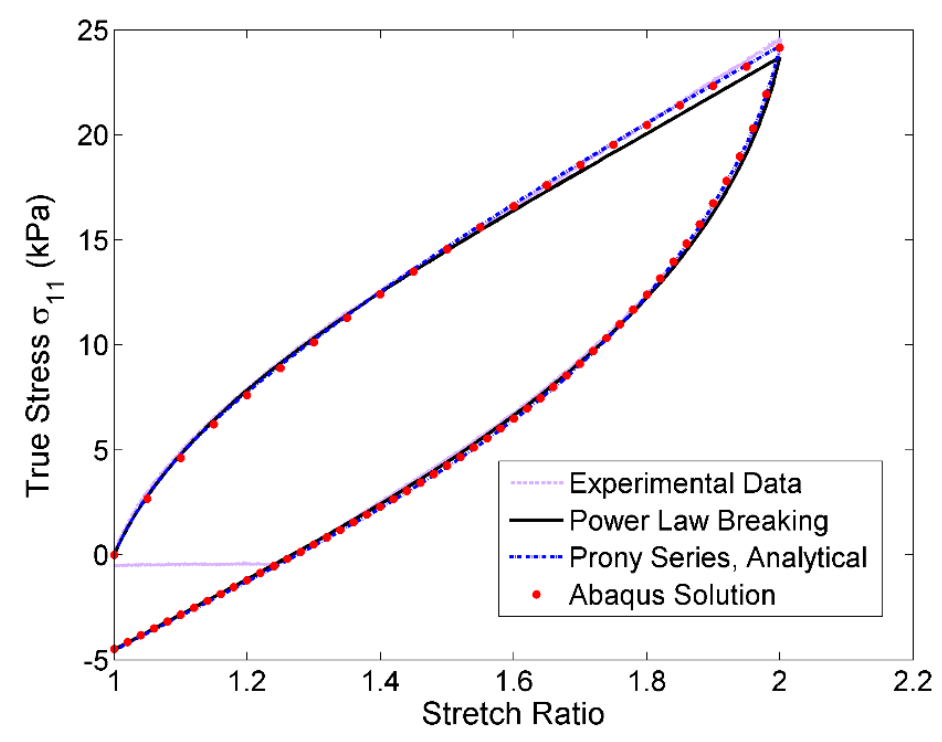

(b)

Figure 10. Comparison of Prony series approximation to power law breaking function. Material parameters are $\mu \rho=2.5414 \mathrm{KPa}, \mu \bar{\gamma}_{\infty}=8.5 \mathrm{KPa} \cdot \mathrm{s}^{-1}, \alpha_{B}=1.6125, t_{B}=0.97 \mathrm{~s}$. (a) Power law breaking function $n(t)$ vs. Prony series approximation. The Prony parameters and characteristic times are: $\tau_{1}=0.3916 s, g_{1}=0.0419 ; \tau_{2}=2.2601 s, g_{2}=0.5681 ; \tau_{3}=22.1507 s, g_{3}=0.3000$ 
; $\tau_{4}=346.2872 s, g_{4}=0.0900$. (b) True stress vs. stretch ratio of a uniaxial tension test. Loading rate is $0.025 \mathrm{~s}^{-1}$, unloading rate is $0.01 \mathrm{~s}^{-1}$, and maximum stretch ratio is $\lambda_{\max }=2.0$

\section{Fracture Simulations and Comparison to Experimental Results}

Results in the previous section show that the FEM implementation of the model is able to accurately capture uniaxial behavior over a range of loading/unloading rates. However, the constitutive model does not provide information on material failure. Further, it is not clear whether it correctly captures the behavior near the crack tip. To check this, a series of fracture tests where samples are loaded at different stretch rates has been performed to measure the load and stretch to failure. We use the simulations to compute the failure loads and compare the results with experimental data. However, these experiments provide only macroscale information. In our gel, the damage is highly localized so we anticipate that the onset of fracture can be characterized by local crack tip field parameters.

Although the stress at the crack tip cannot be measured directly, the crack opening displacement can be measured, and we can compare our FEM simulations to test data. The goal is to demonstrate the ability of the computational model to characterize the behavior of crack tip fields.

\subsection{Validation of 3D Finite Element Procedures Against Neo-Hookean Model}

Theoretical models of fracture typically assume that the crack is loaded under conditions of plane stress or plane strain. In our case, the thickness of the sample is small compared to its lateral dimensions, so plane stress is expected to be a reasonable approximation. However, because of high stress gradient, the plane stress condition may not be satisfied near the crack tip, hence we use a full 3D model in the finite element simulation.

The accuracy of the mesh used in our 3D fracture simulation can be checked by comparing the numerical result with the known asymptotic field at the tip of a Mode I crack in a neo-Hookean solid under plane stress. The geometry of the samples is shown in (Section 2.3, Figure 4, Geometry A). According to Geubelle and Knauss ${ }^{24}$ (also summarized by Long et al. ${ }^{26}$ ), (see Figure 5 for definition of coordinates), the crack opening displacement (COD) is

$$
u_{2}(\theta=\pi)=a \sqrt{r},
$$

and the dominant stress component near the crack tip is:

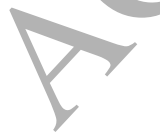

$$
\sigma_{22}(r, \theta=0)=\frac{\mu a^{2}}{4 r}
$$

where $\mu$ is the small strain shear modulus of the Neo-Hookean solid, and $a$ is a parameter dependent on loading and geometry, analogous to a stress intensity factor. 


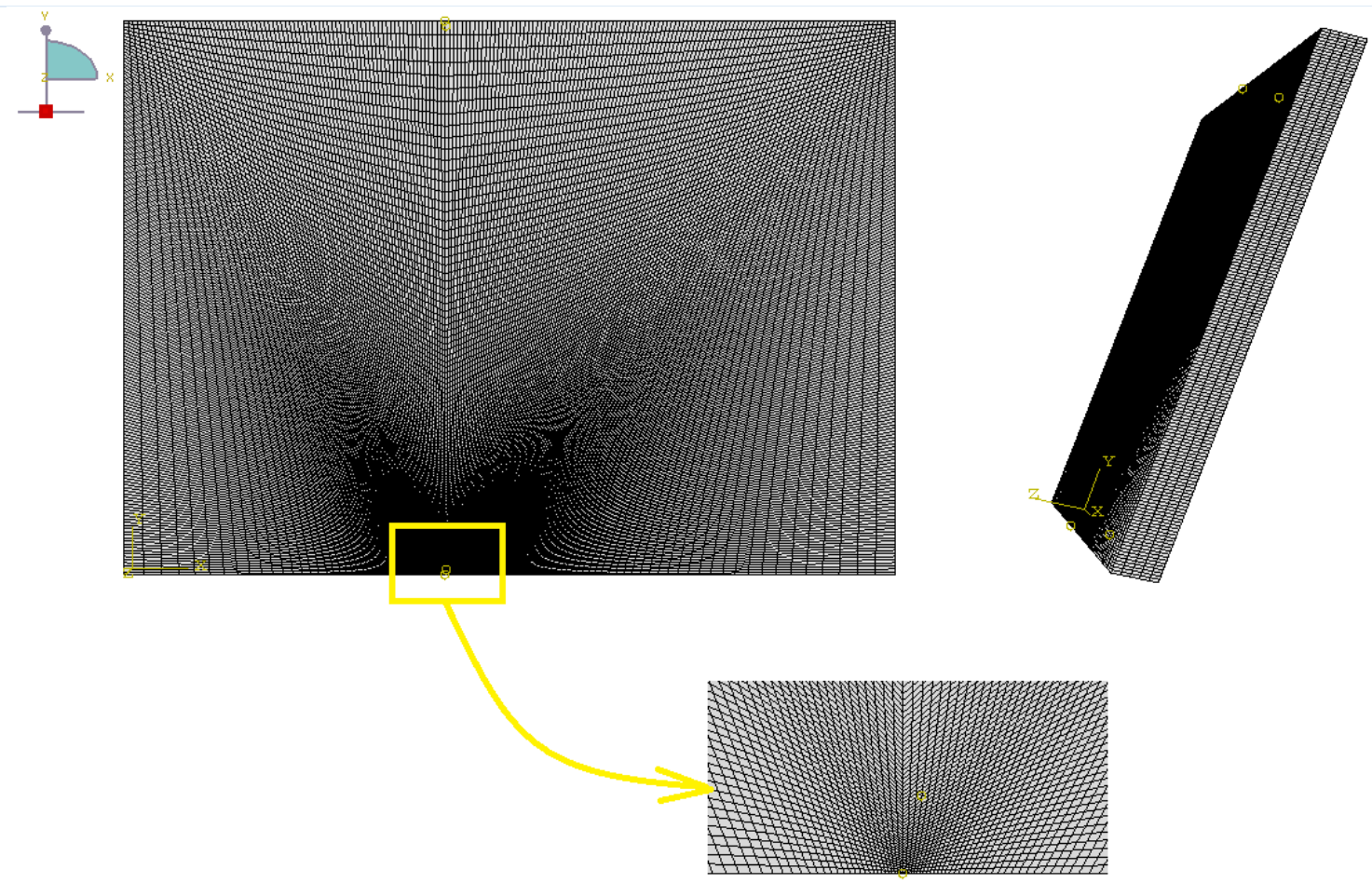

Figure 11. Overview and details of finite element mesh used in validation study. Left figure shows the mesh in xy plane. A biased mesh towards the crack tip (zoom in view at the bottom) is used. For the case of 3D, in the thickness direction ( $z$ direction) the sample uniformly meshed into 5 layers.

We carry out simulations using both 2D plane stress and full 3D geometry, with the mesh biased towards the crack tip as shown in Figure 11. We model the upper half of the crack sample with half its thickness (use symmetric boundary condition), and use a biased mesh towards the crack tip along both $x$ and $y$ axis. Linear elements are used in both 2D and 3D cases (CPS4R for 2D and C3D8H for 3D) and simulations are carried out in ABAQUS Standard. The 2D plane stress FEM is used to check the software's ability to carry out crack tip simulations. A convergence study was performed, varying the crack tip element size in 3D model from $0.003 \mathrm{~mm}$ to $0.1 \mathrm{~mm}$ (sample width $12.5 \mathrm{~mm}$, crack length 5 $\mathrm{mm}$ ) and comparing the computed COD and the dominant true stress $\sigma_{22}$ to theory (see Figure S3 in supplementary information). The COD and the stresses for the 3D model were evaluated at the center line. For each case tested, the displacements and the stresses converge. The CODs are identical, and the true stresses overlap with the theory within 3 elements. So the size of all our crack tip elements in all our simulations are chosen below $0.1 \mathrm{~mm}$. Typical results are shown in Figure 12. Figure 12a shows that both the 2D and 3D model yield very similar COD that are in agreement with the asymptotic result given 
by equation (21). Figure $12 \mathrm{~b}$ shows that the true stress $\sigma_{22}(r, \theta=0)$ directly ahead of the crack tip agrees with equation (22) for the case of plane stress. The 3D finite element results differ from the asymptotic solution very close to the crack tip, due to the fact that the mesh in the thickness direction is coarse. These figures show that the 3D FEM results are in good agreement with the 2D plane stress simulations and with the $2 D$ plane stress asymptotic theory. In the following, all simulations of fracture experiments will be performed in $3 D$.

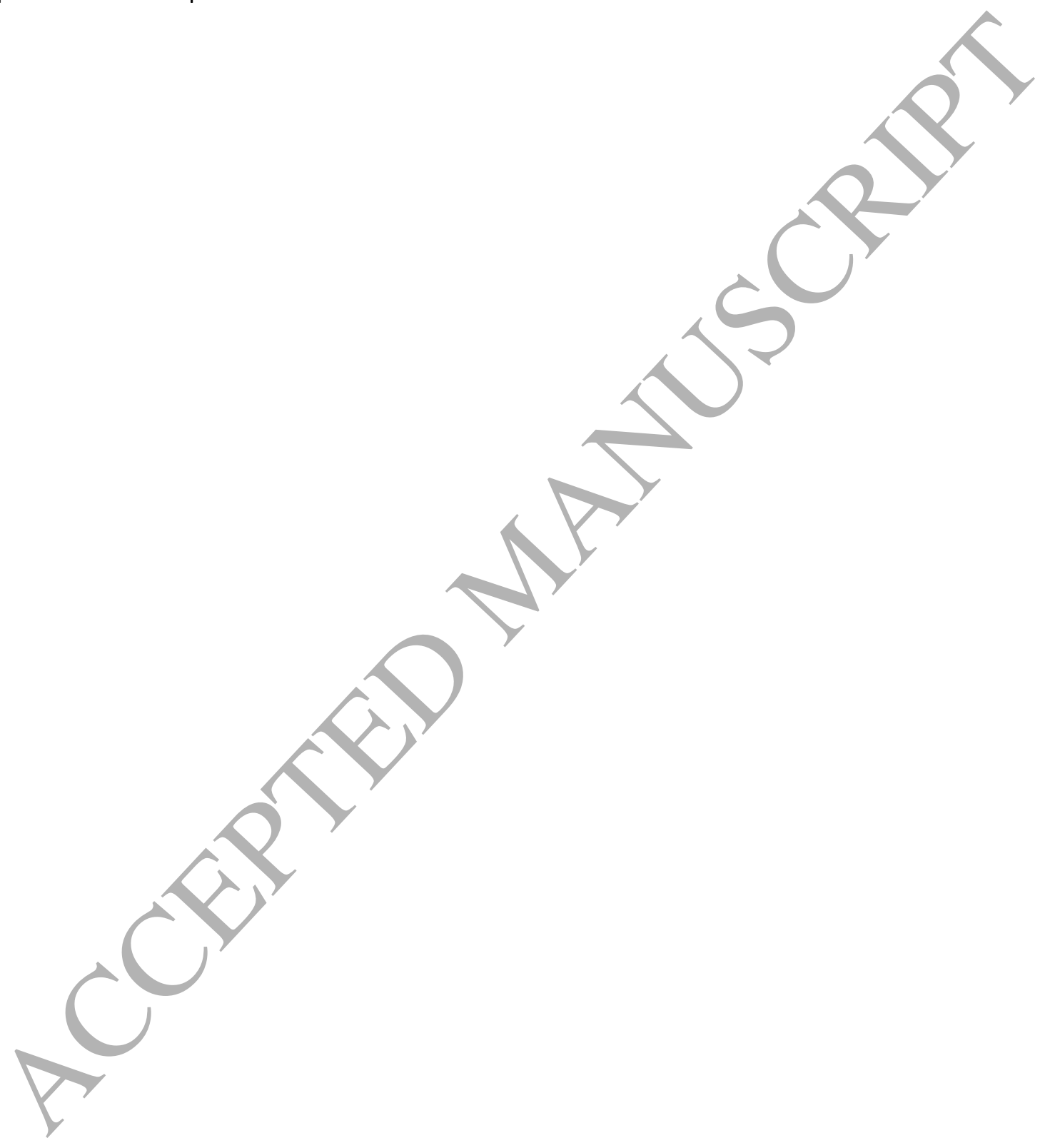




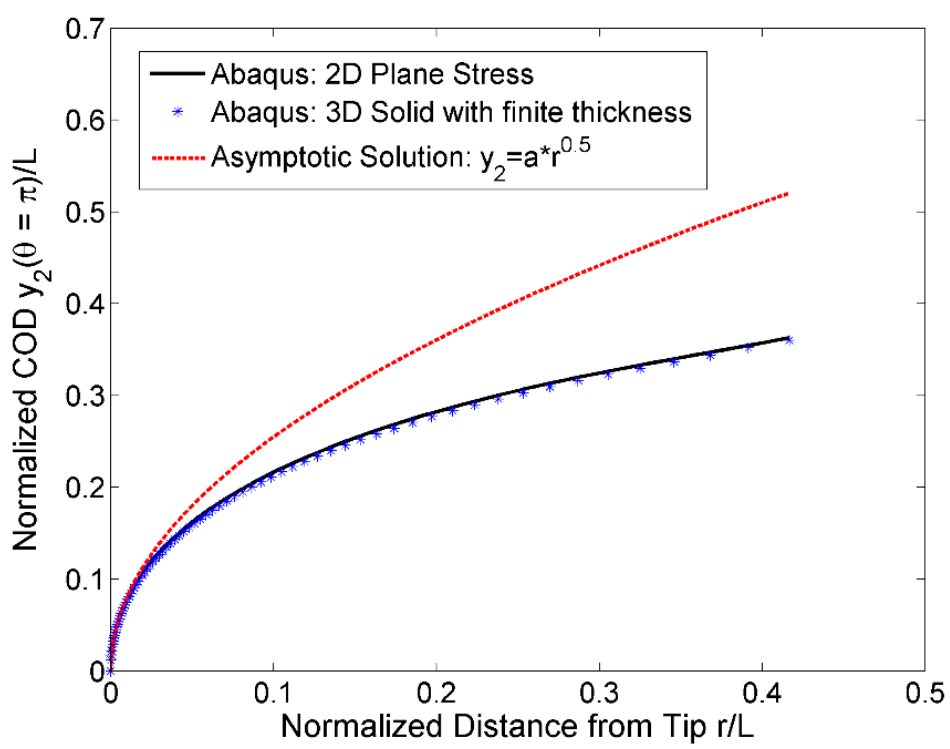

(a)

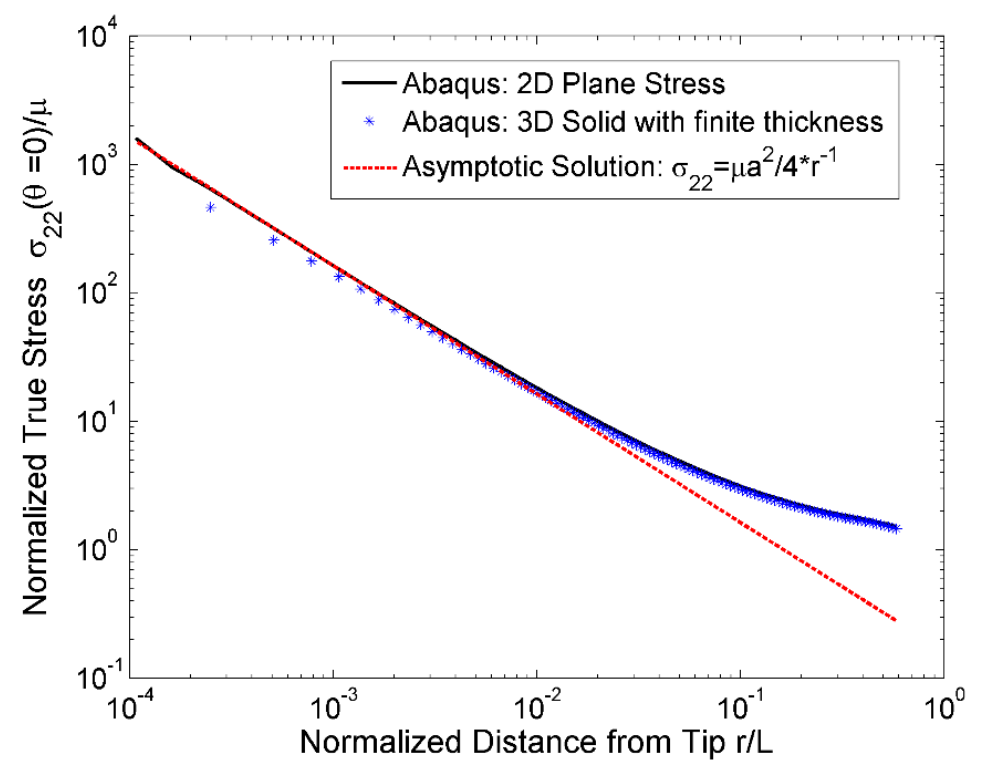

(b)

Figure 12. Comparison of asymptotic plane stress solution with $2 D$ and $3 D$ simulations in $A B A Q U S$ for a Neo-Hookean mode I crack sample. The lengths are normalized by the total width of uncracked sample, $L$, and the stress is normalized by the shear modulus $\mu$. (a) Crack opening displacement; (b) True stress ahead of the crack tip. 3D model has a tip element size 0.003 . 


\subsection{Simulations of constant stretch rate fracture tests}

Experiments were carried out on fracture sample geometries A and B. All samples were loaded to failure and three repeats were performed for all tests. Samples $A$ were loaded at stretch rates of $0.001 / \mathrm{s}, 0.003 / \mathrm{s}, 0.01 / \mathrm{s} 0.03 / \mathrm{s}, 0.1 / \mathrm{s}$ and $0.3 / \mathrm{s}$. One additional set of tests was performed on geometry $B$ at a stretch rate of $0.01 / \mathrm{s}$. In all experiments the load and stretch were measured and recorded up to failure. In all tests, once fracture initiates, the crack propagates rapidly across the specimen.

The material parameters for the simulations of different samples are given in the supplemental information (Table S1). Note that each batch of material has slightly different mechanical response due to the slight difference in chemicals and synthesis procedure when making each batch of samples.

Figure 13 compares the measured and computed nominal stress versus stretch for a single test using sample $A$ at a stretch rate of $\dot{\lambda}=0.01 / s$. It can be seen that the finite element simulation slightly underestimates the load but overall matches the experimental results. It should be noted that beyond the basic material model there are no fitting parameters in all our fracture simulations.

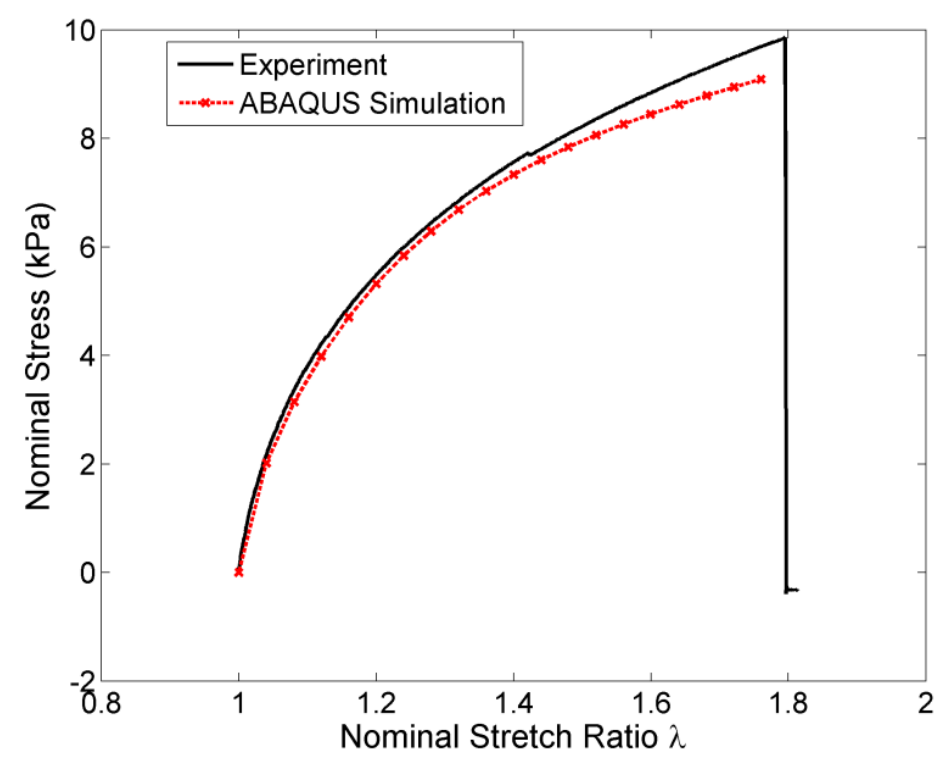

Figure 13. Nominal stress vs. stretch ratio at $0.01 / \mathrm{s}$ loading rate until failure. 


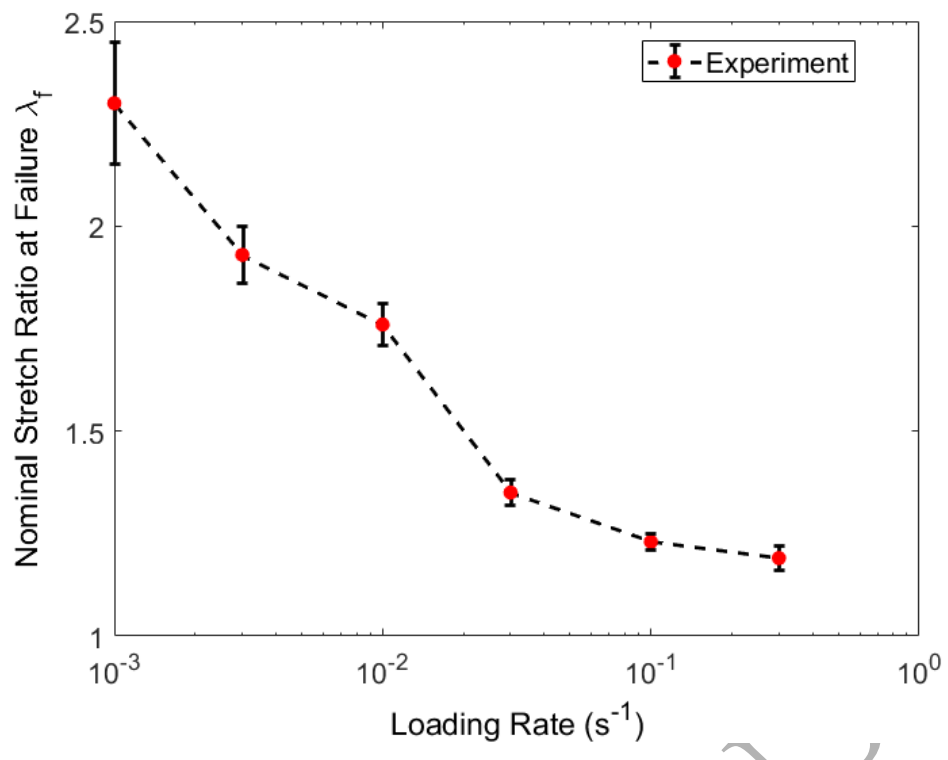

Figure 14. Stretch to failure vs. the loading rate. The vertical bars indicate the range of the experimentally measured stretch to failure. The red dots show the average stretch to failure. Dotted line serves as guide to the eye. These average values were used in the simulations.

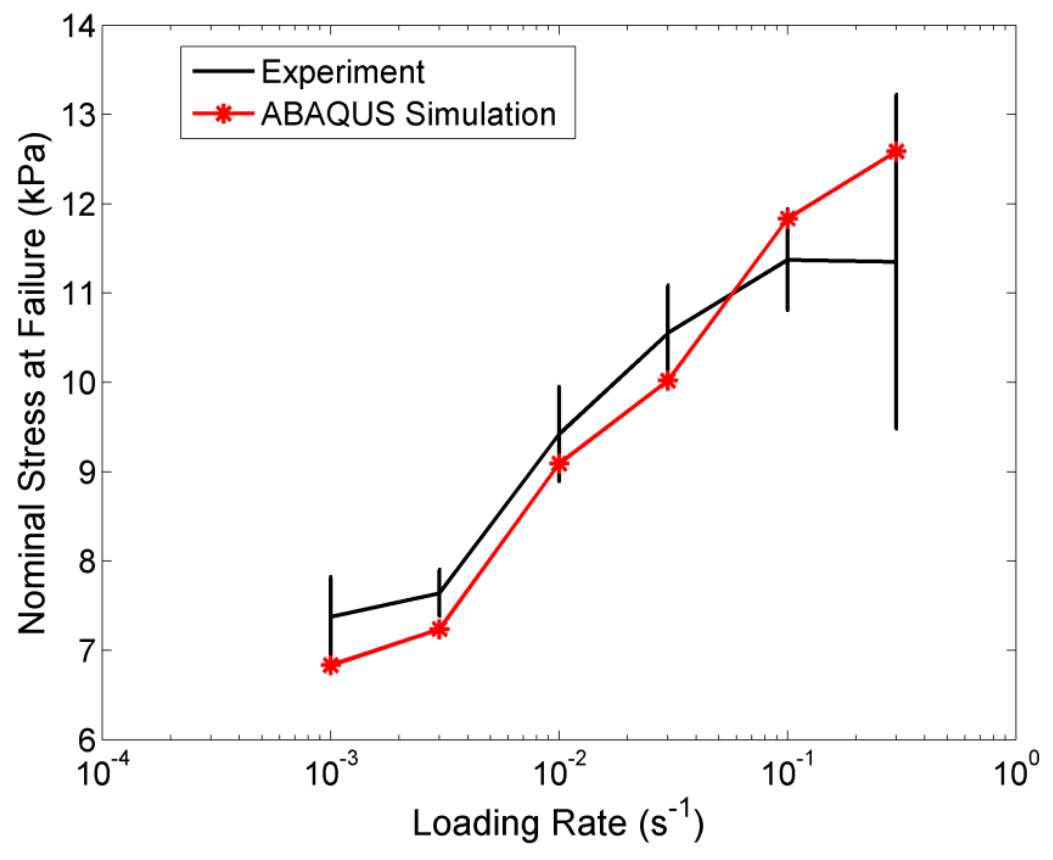

Figure 15. Failure stress (nominal) vs. loading rate. Each simulation is carried out under the given loading rate until the failure stretch is reached. Failure stretch is taken as the average failure stretch (over three tests), shown in Figure 14. 
Results from the fracture experiments are shown in Figure 14 and 15. Figure 14 plots the average stretch to failure in geometry A under different stretch rates. The nominal stress to failure is plotted in Figure 15. Figures 14 and 15 show that with increasing stretch rate, the failure stretch decreases while the nominal failure stress increases. This is because at higher loading rates, the strands between physical crosslinks carry more stress, so when chemical bonds fail, these physical bonds cannot absorb more loads since they are already stretched, as a result, the gel fails at a lower stretch. On the other hand, the failure load increases since the gel is stiffer due to the stresses carried by the physical strands.

We simulate these experiments by stretching the FEM model at the applied stretch rate up to the average of the failure stretches given by the data in Fig. 14. The nominal stress at fracture from these simulations (red symbols) is shown in Figure 15 together with the experimental data (black lines). The computed stress to failure agrees with the experimental data to within the error bars.

These results show that our constitutive model is able to capture the macroscopic response of cracked samples. We now demonstrate that the simulation works equally well at the local level. In the same set of experiments, using a Nikon DSLR camera with a $40 \mathrm{~mm}$ lens, we captured a series of images as the samples were loaded. We then superimposed the crack opening profile from our simulation on top of the images. As figures 16 and 17 show, without exception, the crack profiles predicted by the finite element model fit almost perfectly to those obtained from experiments.

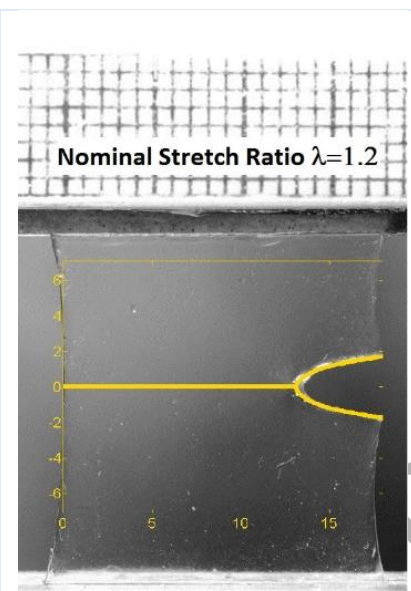

(a)

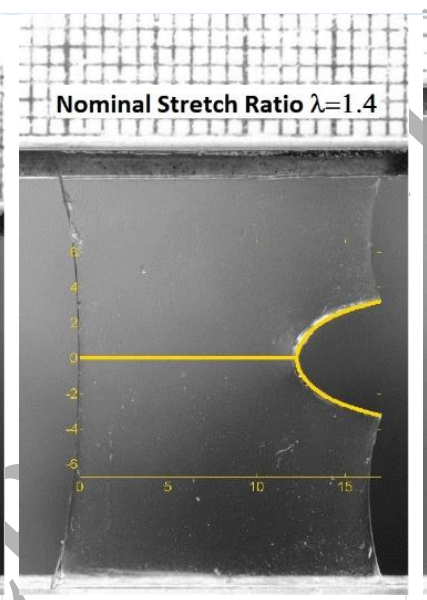

(b)

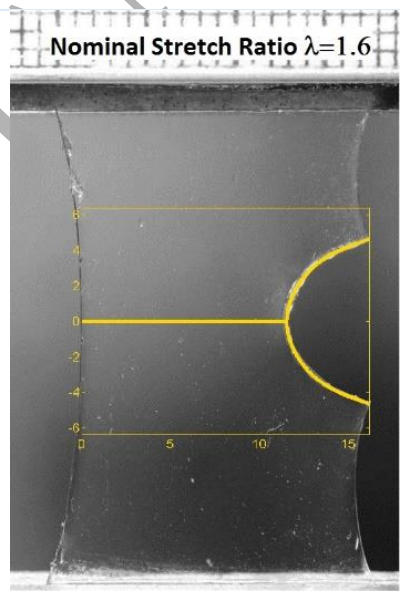

(c)

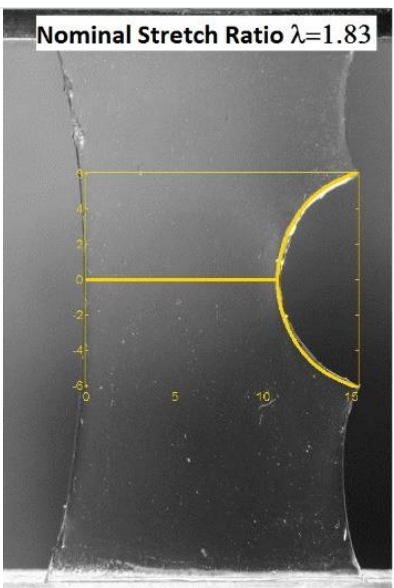

(d)

Figure 16. Crack opening profiles for a cracked sample of geometry $A$ under a loading rate of $0.01 / \mathrm{s}$ and fails at a nominal stretch ratio of 1.83 . The images were taken at nominal stretch ratios of $1.2,1.4,1.6$, 1.83, respectively, and the yellow curves along with the yellow coordinates are the finite element simulation results. 


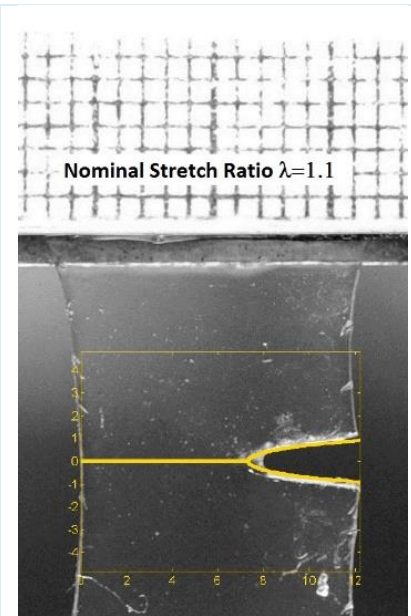

(a)

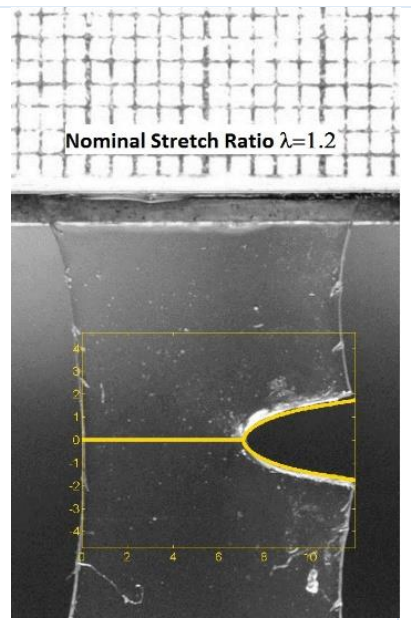

(b)

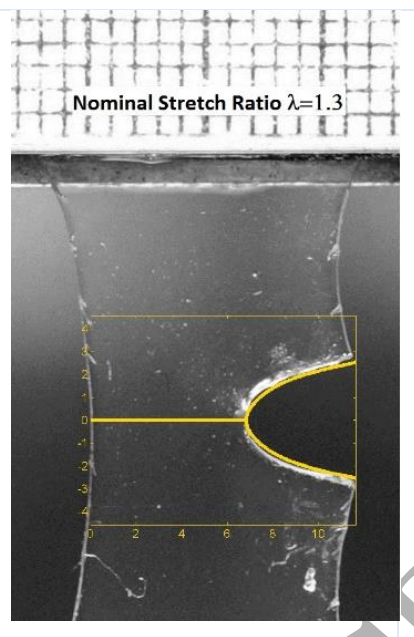

(c)

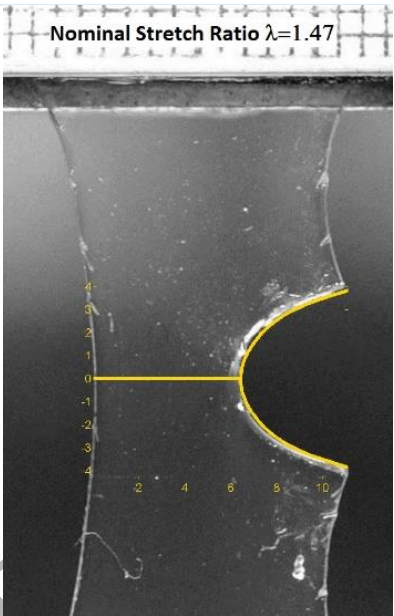

(d)

Figure 17. Crack opening profiles for a cracked sample of geometry $B$ under a loading rate of $0.01 / \mathrm{s}$ and fails at a nominal stretch ratio of 1.47. The images were taken at nominal stretch ratios of 1.1, 1.2, 1.3, 1.47 , respectively, and the yellow curves along with the yellow coordinates are the finite element simulation results.

\section{Summary and Discussion}

Experiments and numerical simulations were carried out to study the time dependent fields in tension loaded, edge cracked specimens made of a dual-crosslink hydrogel. We developed a novel time integration scheme which allows us to bypass the storage problem usually associated with the evaluation of history dependent integrals. We carried out analyses to determine the time dependent crack tip stress fields in a load relaxation test. With no fitting parameters, the 3D finite element simulations are in excellent agreement with experimental data - including the local behavior near the crack tip. The results show that the 3D constitutive model correctly describes the material behavior near the crack tip.

We are surprised at how well our 3D constitutive model based on a neo-Hookean work function is able to capture the opening displacement at the crack tip. It is well known that this model underestimates the amount of strain hardening; therefore, it should overestimate the crack opening displacement. Indeed, in our previous work ${ }^{22}$, we noted that the neo-Hookean model underestimates the nominal stress of un-notched samples loaded at a stretch rate of $0.01 / \mathrm{s}$ when the stretch ratio exceeds 2 . In this previous work, we found that an exponential work function, which reduces to the neo-Hookean model at small strains) works well at all strain levels. A possible explanation is that the material near the crack tip softens due to damage or that the displacement is predicted correctly but not the stresses. In a future work, we plan to implement strain hardening in our numerical model to study the effect of strain hardening on the crack tip fields. 
Crack growth is not studied in this work. The PVA dual crosslinked gels are relatively brittle, and we found that cracks grow quickly once initiated. In previous experiments reported in an earlier paper ${ }^{27}$, we have found it is possible to grow slower cracks provided that the applied nominal stretch rates are very small. However, it must be noted that the gels used in these experiments are made in a different laboratory and more importantly, the experiments are carried out using different sample geometries (the cracks in these experiments are much shorter than those used in this work). We are currently performing these slow crack growth experiments for our samples. Understanding slow crack growth is important since at very slow stretch rates, the stress versus stretch curve of a pure chemical PVA gel and a dual crosslinked PVA gel lies on top of each other until the pure chemical gels fails - typically at a much lower stretch ratio. In our theory, a very slow loading rate implies that the chains between physical crosslinks carry very little or no load, since the loading rate is much smaller than the characteristic breaking time. Here it is important to recall that, in our constitutive model, the stress sustained by a temporary chain is instantaneously relaxed when it breaks, and that immediately after a temporary chain is reattached, it is in a relaxed state and carries no strain energy. The fact that the dual crosslink gel fails at a much higher stretch at very slow loading rate suggested that the physical bonds near the crack tip must bear the load previously carried by broken chemical bonds. Recall that in our theory chemical bonds are not allowed to break. Therefore, these slow crack growth experiments will give valuable information about the load transfer process near the crack tip, which is missing in our constitutive model.

\section{Appendix}

We show that the deformation gradient is time independent by showing that all the field equations as well as the boundary conditions are satisfied by this assumption. Assume the displacement field is independent of time (that is, it is the same as at $t=0+$ ). This will give rise to a spatial varying time independent deformation gradient field. Thus, $\mathbf{F}^{0 \rightarrow t}$ in (1b) is given by $\mathbf{F}\left(\boldsymbol{x}, t=0^{+}\right) \equiv \mathbf{F}^{+}$for all $\mathrm{t}>0$.

This implies that $\mathbf{F}^{\tau \rightarrow t}=\boldsymbol{I}$ at any material point and hence the integral term in (1a) is proportional to $\left(\mathbf{F}^{+}\right)^{-T}$ and can be absorbed into the pressure term. In the paper, this new pressure is denoted by

$$
p^{*} \equiv p-\mu \bar{\gamma}_{\infty} \int_{0}^{t} \phi_{B}\left((t-\tau) / t_{B}\right) d \tau
$$

Thus, the nominal stresses everywhere is given by $\mathbf{P}=-p^{*}\left(\mathbf{F}^{+}\right)^{-T}+\left.2[\rho+n(t)] \frac{d W_{0}}{d I}\right|_{I=I(x, t)} \mathbf{F}^{+}$, which is the relation between an incompressible hyper-elastic solid with a time dependent modulus. Since $\mathbf{F}^{+}$is the exact deformation gradient field at time equal to zero, and $n(t)$ is a function of time, not position, $\mathbf{P}=-p^{*}\left(\mathbf{F}^{+}\right)^{-T}+\left.2[\rho+n(t)] \frac{d W_{0}}{d I}\right|_{I=I(\boldsymbol{x}, t)} \mathbf{F}^{+}$also satisfies the equilibrium equations. The traction free boundary condition on crack faces is clearly satisfied and on the rest of the boundary the displacements are fixed by assumption. Thus, assuming that the solution is unique, the assumption of 
that the deformation gradient at any point is independent of time is valid and the true stresses everywhere is given by (9).

\section{Acknowledgements}

This material is based upon work supported by the National Science Foundation under Grant No. CMMI $-1537087$.

\section{References}

[1] Lee, K. Y.; Mooney, D. J. Hydrogels for tissue engineering. Chem. Rev. 2001, 101 (7), 1869-1879. DOI: $10.1021 / \mathrm{cr} 000108 \mathrm{x}$

[2] Kuo, C. K.; Ma, P. X. lonically crosslinked alginate hydrogels as scaffolds for tissue engineering: part 1. Structure, gelation rate and mechanical properties. Biomaterials 2001, 22, 511-521. DOI: 10.1016/S0142-9612(00)00201-5.

[3] Kwon, H. J.; Yasuda, K.; Gong, J. P.; Ohmiya, Y. Polyelectrolyte hydrogels for replacement and regeneration of biological tissues. Macromol. Res. 2014, 22 (3), 227-235. DOI: 10.1007/s13233-0142045-6.

[4] Qiu, Y.; Park, K. Environment-sensitive hydrogels for drug delivery. Adv. Drug Delivery Rev. 2001, 53, 321-339. DOI: 10.1016/S0169-409X(01)00203-4

[5] Gong, J. P.; Katsuyama, Y.; Kurokawa, T.; Osada, Y. Doúble-network hydrogels with extremely high mechanical strength. Adv. Mater. 2003, 15 (14), 1155-1158. DOI: 10.1002/adma.200304907.

[6] Webber, R. E.; Creton, C.; Brown, H. R.; Gong, J. P. Large Strain Hysteresis and Mullins effect of tough Double-Network Hydrogels. Macromolecules 2007, 40 (8), 2919-2927. DOI: 10.1021/ma062924y.

[7] Nakajima, T.; Kurokawa, T.; Ahmed, S.; Wu, W.; Gong, J. P. Characterization of internal fracture process of double network hydrogels under uniaxial elongation. Soft Matter 2013, 9 (6), 1955-1966. DOI: 10.1039/C2SM27232F.

[8] Brown, H. R. A model of the fracture of double network gels. Macromolecules 2007, 40 (10), 3815-3818. DOI: 10.1021/ma062642y.

[9] Gong, J.P. Why are double network hydrogels so tough? Soft Matter 2010, 6 (12), 2583-2590. DOI: 10.1039/B924290B.

[10] Wang, X.; Hong, W. Pseudo-elasticity of a double network gel. Soft Matter 2011, 7 (18), 8576-8581. DOI: 10.1039/C1SM05787A.

[11] Henderson, K. J.; Zhou, T. C.; Otim, K. J.; Shull, K. R. Ionically cross-linked triblock copolymer hydrogels with high strength. Macromolecules 2010, 43 (14), 6193-6201. DOI: 10.1021/ma100963m. 
[12] Lin, W. C.; Fan, W.; Marcellan, A.; Hourdet, D.; Creton, C. Large strain and fracture properties of poly (dimethylacrylamide)/silica hybrid hydrogels. Macromolecules 2010, 43 (5), 2554-2563. DOI: 10.1021/ma901937r.

[13] Sun, J. Y.; Zhao, X.; Illeperuma, W. R.; Chaudhuri, O.; Oh, K. H.; Mooney, D. J.; Vlassak, J. J.; Suo, Z. Highly stretchable and tough hydrogels. Nature 2012, 489, 133-136. DOI: 10.1038/nature11409.

[14] Sun, T. L.; Kurokawa, T.; Kuroda, S.; Ihsan, A. B.; Akasaki, I.; Sato, K.; Haque, M. A.; Nakajima, T.; Gong, J. P. Physical hydrogels composed of polyampholytes demonstrate high toughness and viscoelasticity. Nat. Mater. 2013, 12, 932-937. DOI: 10.1038/nmat3713.

[15] Mayumi, K.; Marcellan, A.; Ducouret, G.; Creton, C.; Narita, T. Stress-Strain Relationship of Highly Stretchable Dual Cross-Link Gels: Separability of Strain and Time Effect. ACS Macro Lett. 2013, 2 (12), 1065-1068. DOI: 10.1021/mz4005106.

[16] Narita, T.; Mayumi, K.; Ducouret, G.; Hebraud, P. Viscoelastic Properties of Poly(vinyl alcohol) Hydrogels Having Permanent and Transient Cross-Links Studied by Microrheology, Classical Rheometry, and Dynamic Light Scattering. Macromolecules 2013, 46 (10), 4174-4183. DOI: 10.1021/ma400600f.

[17] Luo, F.; Sun, T. L.; Nakajima, T.; Kurokawa, T.; Zhao, Y.; Sato, K.; Ihsan, A. B.; Li, X.; Guo, H.; Gong, J. P. Oppositely Charged Polyelectrolytes Form Tough, Self-Healing, and Rebuildable Hydrogels. Advanced Materials 2015, 27, 2722-2727. DOI: 10.1002/adma.201500140.

[18] Rose, S.; Dizeux, A.; Narita, T.; Hourdet, D.; Marcellan, A. Time Dependence of Dissipative and Recovery Processes in Nanohybrid Hydrogels. Macromolecules 2013, 46(10), 4095-4104. DOI: 10.1021/ma400447j.

[19] Long, R.; Mayumi, K.; Creton, C.; Narita, T.; Hui, C. Y. Time dependent behavior of a dual cross-link self-healing gel: theory and experiments. Macromolecules 2014, 47 (20), 7243-7250. DOI:

$10.1021 / \mathrm{ma501290h}$.

[20] Long, R.; Mayumi, K.; Creton, C.; Narita, T.; Hui, CY. Rheology of a dual crosslink self-healing gel: theory and measurement using parallel-plate torsional rheometry. J. Rheol. 2015, 59, 643-665. DOI: 10.1122/1.4915275.

[21] Guo, J.; Long, R.; Mayumi, K.; Hui, C. Y. Mechanics of a Dual Cross-Link Gel with Dynamic Bonds: Steady State Kinetics and Large Deformation Effects. Macromolecules 2016, 49 (9), 3497-3507. DOI: 10.1021/acs.macromol.6b00421.

[22] Bilici, C.; Ide S.; Okay, O. Yielding Behavior of Tough Semicrystalline Hydrogels. Macromolecules 2017, 50(9), 3647-3654. DOI: 10.1021/acs.macromol.7b00507.

[23] Li, J.; Suo, Z.; Vlassak, J. J. Stiff, strong, and tough hydrogels with good chemical stability. J. Mater. Chem. B 2014, 2(39), 6708-6713.

[24] Geubelle, P.H.; Knauss, W.G. Finite strain at the tip of a crack in a sheet of hyperelastic materials: I. Homogeneous case, J. Elast. 35 (1994) 61-98. DOI: 10.1007/BF00115539. 
[25] ABAQUS. Finite-strain viscoelasticity. Abaqus 2016 Documentation.

[26] Long, R.; Hui, C. Y. Crack tip fields in soft elastic solids subjected to large quasi-static deformation a review. Extreme Mechanics Letters 2015 (4), 131-155. DOI: 10.1016/j.eml.2015.06.002.

[27] Mayumi, K.; Guo, J.; Narita, T.; Hui, C. Y.; Creton, C. Fracture of dual crosslink gels with permanent and transient crosslinks. Extreme Mechanics Letters 2016 (6), 52-59. DOI: 10.1016/j.eml.2015.12.002.

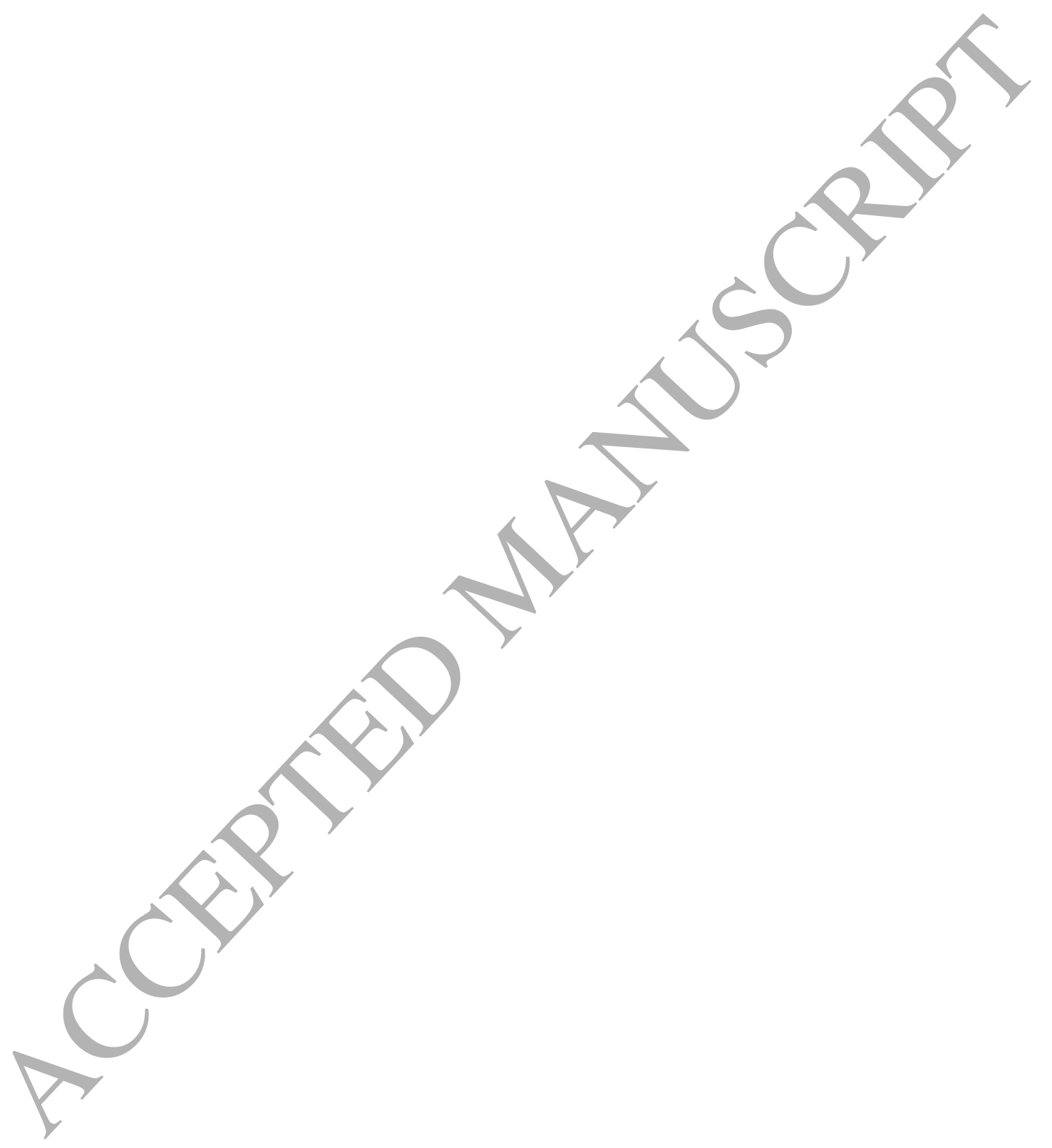

